\title{
Singular boundary method for wave propagation analysis in periodic structures
}

\author{
Zhuojia Fu ${ }^{1,2 *}$, Wen Chen ${ }^{1,2}$, Pihua Wen ${ }^{3}$, Chuanzeng Zhang ${ }^{4}$ \\ 1. Center for Numerical Simulation Software in Engineering and Sciences, College of Mechanics and \\ Materials, Hohai University, Nanjing, Jiangsu, 211100, China \\ 2. State Key Laboratory of Acoustics, Institute of Acoustics, Chinese Academy of Sciences, Beijing \\ 100190, China \\ 3. School of Engineering and Materials Science, Queen Mary, University of London, London E1 4NS, \\ $U K$ \\ 4. Department of Civil Engineering, University of Siegen, Paul-Bonatz-Str. 9-11, D-57076 Siegen, \\ Germany
}

\begin{abstract}
A strong-form boundary collocation method, the singular boundary method (SBM), is developed in this paper for the wave propagation analysis in periodic structures. The SBM is of several advantages including mathematically simple, easy-to-program, meshless with the application of the concept of origin intensity factors in order to eliminate the singularity of the fundamental solutions and avoid the numerical evaluation of the singular integrals in the boundary element method. Due to the periodic behaviors of the structures, the SBM coefficient matrix can be represented as a block Toeplitz matrix. By employing three different fast Toeplitz-matrix solvers, the computational time and storage requirements are significantly reduced in the proposed SBM analysis. To demonstrate the effectiveness of the proposed SBM formulation for wave propagation analysis in periodic structures, several benchmark examples with a non-dimensional wavenumber $k d<60$ are presented and discussed, where $k$ is the wavenumber and $d$ is the maximum diameter of the periodic structures. The proposed SBM results are compared with the analytical solutions, the reference results and the COMSOL software.
\end{abstract}

Keywords: Singular boundary method, periodic structures, Toeplitz matrix, wave propagation, meshless method, Fast Fourier Transform

\section{Introduction}

The control of wave propagation in periodic structures [1-3] is of great importance in the design and manufacture of modern acoustic and optical devices, such as photonic crystals, photovoltaic devices and metamaterials [4,5]. To improve the performance of these devices, various numerical methods have been proposed to simulate and manipulate wave propagation in periodic structures, such as the transfer 
matrix method [6], multiple scattering theory method [7], finite element method (FEM) [8], boundary element method (BEM) [9], plane wave expansion method (PWEM) [10], method of fundamental solutions (MFS) [11], to mention just a few of them.

Among the above-mentioned numerical methods, the transfer matrix method is mainly implemented to $1 \mathrm{D}$ periodic structures. The multiple scattering theory method is limited to solving 3D periodic structures with special scatterers (sphere or cylinder). The domain-discretization FEM requires the additional treatments to carefully deal with the exterior domain of the periodic structures. The BEM [12] requires only boundary discretization, and introduces the fundamental solution as its basis function to satisfy the governing equation and the Sommerfeld radiation condition at infinity in advance. However, it is a sophisticated mathematical and time-consuming issue for numerical integration over the singularities in the BEM. Besides, both the PWEM and MFS can avoid these troublesome issues appeared in the BEM due to the use of the nonsingular plane wave basis function or the placement of the source nodes away from the real physical boundary. However, both the PWEM and MFS provide the highly ill-conditioning resultant matrices with moderate node number, which may jeopardize the accuracy of their numerical solutions. In addition, the PWEM has a relatively slow convergence rate. In the MFS, the determination of the efficient placement of the source nodes is vital for numerical accuracy and reliability, and it requires an additional computational cost.

Based on the merits and demerits of the above-mentioned numerical methods, a meshless boundary collocation method, the singular boundary method (SBM), has been proposed by Chen et al. in 2009. The SBM [13,14] employs the fundamental solutions as the basis functions, and introduces the concept of "origin intensity factors" (source intensity factors) to take the place of the singularities encountered in the fundamental solutions at origin. It inherits the merits from the BEM and the MFS, and avoids the numerical computation of the singular integrals in the BEM, and circumvents the troublesome placement of the source nodes in the MFS. In addition, unlike the other boundary collocation methods (MFS and PWEM), the resultant 
matrix obtained by the SBM has the reasonable condition number as same as the BEM.

It should be mentioned that the determination of the origin intensity factors (OIFs) is one of the key issues in the SBM implementation. So far, there are four approaches $[15,16]$ proposed to determine the OIFs of both the fundamental solutions and their derivatives. The efficiency and accuracy of the SBM have been verified for the potential problems, acoustic and elastic waves, and water waves with arbitrary complex-shaped geometries. Table 1 lists the conclusion for the comparisons between these four approaches in the SBM, where SLE denotes the system of linear equations. In the table, the more "**" it has, the better it is.

Table 1. The comparisons between these four approaches in the SBM

\begin{tabular}{ccccc}
\hline & Approach1 & Approach2 & Approach3 & Approach4 \\
\hline Accuracy & $* *$ & $* * * *$ & $*$ & $* * *$ \\
Stability & $*$ & $* * *$ & $* * * *$ & $* * * *$ \\
Easy to use & $*$ & $* *$ & $* * *$ & $* * * *$ \\
& Inner sample & Boundary sample & Lowest accuracy, & \\
Inconvenience & node, Solve SLE & node, Solve SLE & Numerical & $/$ \\
& twice & twice & Integral & \\
\hline
\end{tabular}

Besides, the SBM resultant matrix is very large and dense similar to the BEM. It is necessary to combine the SBM with the additional techniques to alleviate the computational and storage requirements for large-scale applications, such as the fast multipole methods [17,18], the adaptive cross approximation [19], the Fast Fourier Transform (FFT) [20, 21] and the pre-corrected Fast Fourier Transform (pFFT) [22, 23]. It has been shown that the SBM formulation for the periodic structures leads to a Toeplitz-type matrix similar to the BEM [24]. Therefore, several efficient and fast algorithms can be used to accelerate the computation of the SBM with the special structure of Toeplitz matrix (constant elements along each diagonal). Levinson [25] derived an $O\left(n^{2}\right)$ algorithm for a $n \times n$ Toeplitz matrix. Chandrasekaran and Sayed [26] proposed a stable fast solver for non-symmetric Toeplitz-type matrices. Chan [27] introduced the iterative solvers for Toeplitz matrices. Ferreira and Dominguez [28] proposed an $O(n \log n)$ algorithm, which extends a Toeplitz matrix 
to a circulant matrix by adding more equations, and implements the simple iterative algorithm in conjunction with the Fast Fourier Transform (FFT) to obtain the solution of Toeplitz matrix. Karimi et al. [29] introduced the algorithm developed by Ferreira and Dominguez into the BEM for acoustic wave analysis in periodic structures.

In this study, the SBM in conjunction with Fast Toeplitz-type Matrix Solvers (FTMS) is introduced to wave propagation analysis in periodic structures. In the SBM implementation, the Approach4, the empirical formula coupled with the subtracting and adding-back technique [15], is used to determine the origin intensity factors. In addition, three fast Toeplitz-type matrix solvers are implemented according to the types of the SBM resultant matrix. The paper is divided into the following sections. In Section 2, a detailed numerical implementation of the proposed SBM-FTMS model is described. Section 3 provides some numerical examples with a non-dimensional wavenumber $k d<60$ to demonstrate the effectiveness of the proposed method. Finally, in Section 4 some conclusions are drawn from the present analysis.

\section{Methodology}

\subsection{Time-harmonic wave propagation model}

Consider the time-harmonic wave propagation in a homogeneous and isotropic medium $D$ exterior to the closed bounded curve $\Gamma$ of the periodic structures, which is described by the Helmholtz equation

$$
\nabla^{2} u(x)+k^{2} u(x)=0, \quad x \in D,
$$

subjected to the boundary conditions

$$
\begin{gathered}
u(x)=\bar{u} \quad x \in \Gamma_{D}, \\
q(x)=\frac{\partial u(x)}{\partial \mathbf{n}}=\bar{q} \quad x \in \Gamma_{N},
\end{gathered}
$$

where $k=\omega / c$ is the wavenumber, $\omega$ the angular frequency, $c$ the wave speed in the exterior medium $D$, and $\mathbf{n}$ the outward unit normal on the physical boundary. $\Gamma_{D}$ and $\Gamma_{N}$ represent the boundaries with the essential boundary (Dirichlet) and the natural boundary (Neumann) conditions, respectively, which construct the whole 
closed bounded curve $\Gamma=\Gamma_{D}+\Gamma_{N}$, and $u$ is the complex-valued amplitude of the displacement, velocity potential or wave pressure. For the exterior wave propagation analysis, it should be accomplished by imposing an appropriate radiation condition at infinity, which is termed as the Sommerfeld radiation condition

$$
\lim _{r \rightarrow \infty} r^{\frac{1}{2}(\operatorname{dim}-1)}\left(\frac{\partial u}{\partial r}-i k u\right)=0
$$

where dim is the dimension of the wave propagation problems, and $i=\sqrt{-1}$.

\subsection{Singular boundary method}

For solving the time-harmonic wave propagation problems (1)-(2), the SBM approximate solutions $u(x)$ and $q(x)$ can be expressed by utilizing a linear combination of the Helmholtz fundamental solutions as follows

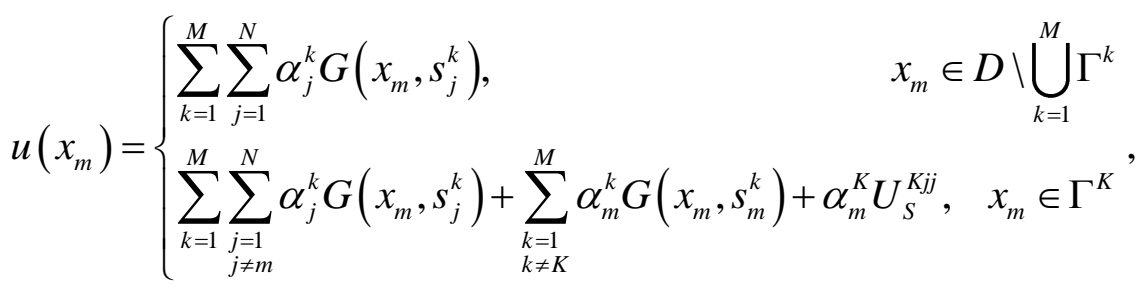

$$
\begin{aligned}
& q\left(x_{m}\right)= \begin{cases}\sum_{k=1}^{M} \sum_{j=1}^{N} \alpha_{j}^{k} \frac{\partial G\left(x_{m}, s_{j}^{k}\right)}{\partial \mathbf{n}_{x}}, & x_{m} \in D \backslash \bigcup_{k=1}^{M} \Gamma^{k} \\
\sum_{\substack { k=1 \\
\begin{subarray}{c}{j \\
j \neq m{ k = 1 \\
\begin{subarray} { c } { j \\
j \neq m } }\end{subarray}}^{N} \alpha_{j}^{k} \frac{\partial G\left(x_{m}, s_{j}^{k}\right)}{\partial \mathbf{n}_{x}} & , \quad x_{m} \in \Gamma^{K}, \\
+\sum_{\substack{k=1 \\
k \neq K}}^{M} \alpha_{m}^{k} \frac{\partial G\left(x_{m}, s_{m}^{k}\right)}{\partial \mathbf{n}_{x}}+\alpha_{m}^{K} Q_{S}^{K j j} & \end{cases}
\end{aligned}
$$

where $M$ is the number of the scatterers in the periodic structures, $N$ denotes the number of source points $s_{j}^{k}$ on the $k$ th scatterer, $\alpha_{j}^{k}$ represents the $j$ th unknown coefficient of the $k$ th scatter, $\mathbf{n}_{x}$ is the outward unit normal vector on the collocation points $x_{m}, \mathrm{G}\left(x_{m}, s_{j}^{k}\right)=\left\{\begin{array}{l}i H_{0}\left(k r_{m j}\right) / 4, \operatorname{dim}=2 \\ e^{i k r_{m j}} /\left(4 \pi r_{m j}\right), \operatorname{dim}=3\end{array}\right.$ are the Helmholtz fundamental

solutions, $H_{n}$ is the $n$th order Hankel function of the first kind, and $r_{m j}=\left\|x_{m}-s_{j}^{k}\right\|_{2}$ 
is the Euclidean distance. If the collocation points and source points coincide, i.e., $x_{m}=s_{j}^{k}$, we have the well-known singularities in the Helmholtz fundamental solutions. The SBM introduces the concept of the origin intensity factors $U_{S}^{K j j}$ and $Q_{S}^{K j j}$ to take the place of these singularities. Due to the periodic structures, each scatterer has the same geometry with the same set of boundary nodes in this study. Therefore, the origin intensity factors $U_{S}^{K j j}$ and $Q_{S}^{K j j}$ for each scatterer are the same, namely, $U_{S}^{K j j}=U_{S}^{j j}$ and $Q_{S}^{K j j}=Q_{S}^{j j}, K=1,2, \cdots, M$. According to the same order of the singularities in the Laplace and Helmholtz fundamental solutions [14], the origin intensity factors $U_{S}^{j j}$ and $Q_{S}^{j j}$ can be represented as follows

$$
\begin{aligned}
& U_{S}^{j j}=U_{S 0}^{j j}+B, \quad x_{m}=s_{j}^{k}, \\
& Q_{S}^{j j}=Q_{S 0}^{j j}, \quad x_{m}=s_{j}^{k},
\end{aligned}
$$

where $U_{S 0}^{j j}$ and $Q_{S 0}^{j j}$ are the OIFs of the corresponding Laplace equation,

$$
B= \begin{cases}-(\ln (k / 2)+\gamma-i \pi / 2) /(2 \pi), & \operatorname{dim}=2, \\ i k /(4 \pi), & \operatorname{dim}=3,\end{cases}
$$

and $\gamma=0.57721566490153286 \cdots$ is the Euler constant. In this paper, we implement the Approach4 based on the empirical formula and the subtracting and adding-back technique to determine the origin intensity factors $U_{S 0}^{j j}$ and $Q_{S 0}^{j j}$, which can be represented as follows

$$
\begin{gathered}
U_{S 0}^{j j}=\left\{\begin{array}{ll}
-\frac{1}{2 \pi} \ln \left(\frac{L_{j}}{2 \pi}\right), & \operatorname{dim}=2 \\
\frac{1}{4 \pi}\left(\frac{\pi^{4}}{25 \sqrt{L_{j}}}+\frac{(\ln (\pi))^{2}}{L_{S}}\right), & \operatorname{dim}=3
\end{array},\right. \\
Q_{S 0}^{j j}=\frac{1}{L_{m}}-\sum_{\substack{j=1 \\
j \neq m}}^{N} \frac{L_{j}}{L_{m}} \frac{\partial G_{0}\left(x_{m}, s_{j}\right)}{\partial \mathbf{n}_{s}},
\end{gathered}
$$

where the Laplace fundamental solutions are given by $G_{0}=\left\{\begin{array}{ll}-\ln \left(r_{m j}\right) /(2 \pi), & \operatorname{dim}=2 \\ 1 /\left(4 \pi r_{m j}\right), & \operatorname{dim}=3\end{array}\right.$, 
$\mathbf{n}_{s}$ is the outward unit normal vector on the source points $s_{j}, L_{S}$ denotes the surface area of the $3 \mathrm{D}$ object, and $L_{j}$ is half-length of the curve $s_{j-1} s_{j+1}$ between the source points $s_{j-1}$ and $s_{j+1}$ for 2D problems or the infinitesimal area of the source point $s_{j}$ for 3D problems as shown in Fig. 1. More details about the determination of the OIFs can be found in the literatures $[15,16]$.

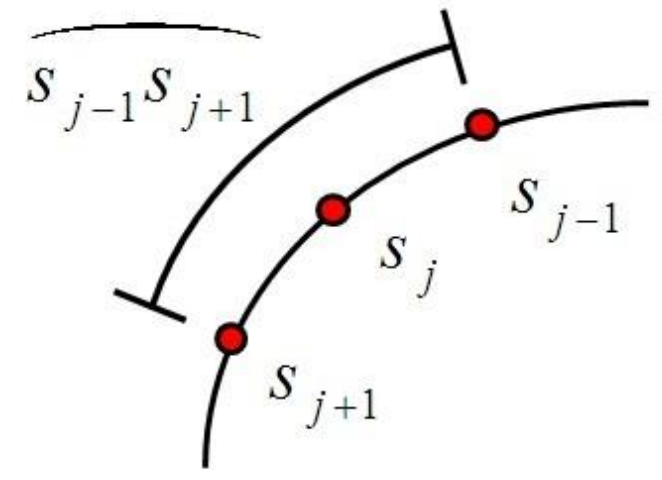

(a)

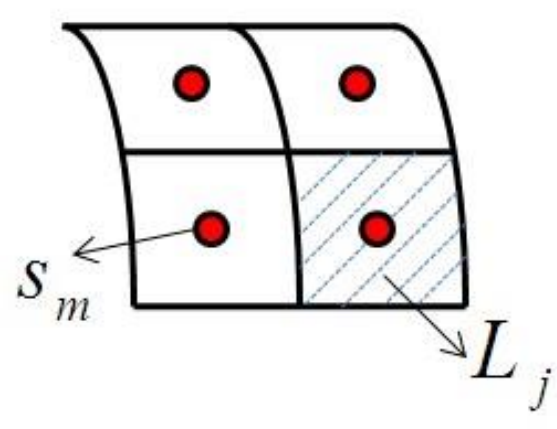

(b)

Fig. 1 The schematic configuration of (a) the source points $s_{j}$ and the curve $s_{j-1} s_{j+1}$ for $2 \mathrm{D}$ problems and (b) the source points $s_{j}$ and the corresponding infinitesimal area $L_{j}$ for $3 \mathrm{D}$ problems.

By applying either Eq. (3a) or (3b) at all the boundary collocation points on the physical boundaries of the periodic structures, the following linear system of equations can be obtained by

$$
\mathbf{A \alpha}=\mathbf{b},
$$

where $\mathbf{A}$ is the SBM coefficient matrix, $\boldsymbol{\alpha}$ and $\mathbf{b}$ denote the unknown coefficient vector and the known boundary condition vector, respectively. After determining the vector $\boldsymbol{\alpha}$, the displacement, velocity potential or wave pressure at any point inside the domain or on the boundary can be evaluated via Eqs. (3a) and (3b).

\subsection{Fast Toeplitz-type matrix solver}

In this section, three different fast Toeplitz-type matrix solvers (FTMS) have been employed to the present SBM simulation according to the types of periodic soft/rigid structures. 


\section{(a) Type 1: Axisymmetric structure}

For solving the axisymmetric structure problems, the SBM coefficient matrix A is a block circulant matrix $\mathbf{C}$ with the form

$$
\mathbf{A}=\mathbf{C}=\left(\begin{array}{cccc}
\mathbf{C}_{0} & \mathbf{C}_{1} & \cdots & \mathbf{C}_{M-1} \\
\mathbf{C}_{M-1} & \mathbf{C}_{0} & \ddots & \mathbf{C}_{M-2} \\
\cdots & \ddots & \ddots & \vdots \\
\mathbf{C}_{1} & \mathbf{C}_{2} & \cdots & \mathbf{C}_{0}
\end{array}\right)
$$

where $\mathbf{C}_{i}$ is an $N \times N$ matrix. The number of the unique blocks $M$ corresponds to the number of the scatterers $M$ in the periodic structures. Fig. 2 shows a typical axisymmetric structure with top-shaped section [30], whose surface can be represented as

$\Gamma=\left\{\left(x_{1}, x_{2}, x_{3}\right): x_{1}=R \cos \phi \cos \varphi, x_{2}=R \cos \phi \sin \varphi, x_{3}=R \sin \phi, 0 \leq \varphi \leq 2 \pi,-\frac{\pi}{2} \leq \phi \leq \frac{\pi}{2}\right\}$, in which $R(\phi, \varphi)=0.144(1+0.3 \cos (4 \phi))$.

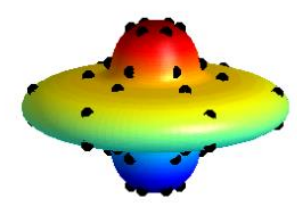

Fig. 2 Schematic configuration of top-shaped axisymmetric structure

According to the property of the block circulant matrix, only the first block row $\mathbf{R}=\left[\begin{array}{llll}\mathbf{C}_{0} & \mathbf{C}_{1} & \cdots & \mathbf{C}_{M-1}\end{array}\right]$ of block circulant matrix $\mathbf{C}$ needs to be stored and computed. Thus, the computational storage can be reduced to $1 / M$ storage of the full coefficient matrix $\mathbf{C}$. Moreover, the inverse matrix $\mathbf{C}^{-1}$ can be fast calculated by using the Fast Fourier Transform (FFT). In the FFT implementation, a block Fourier matrix $\quad \mathbf{F}_{B}$ is defined as the following $p N \times p N$ square matrix

$$
\mathbf{F}_{B}=\mathbf{F} \otimes \mathbf{I}_{N},
$$


where $\mathbf{I}_{N}$ is an $N \times N$ identity matrix, $\otimes$ the Kronecker product, and the Fourier matrix $\mathbf{F}$ is the following $p \times p$ square matrix

$$
\mathbf{F}=\frac{1}{\sqrt{p}}\left(\begin{array}{ccccc}
1 & 1 & 1 & \cdots & 1 \\
1 & \varepsilon_{1} & \varepsilon_{2} & \cdots & \varepsilon_{p-1} \\
1 & \varepsilon_{1}^{2} & \varepsilon_{2}^{2} & \cdots & \varepsilon_{p-1}^{2} \\
\cdots & \cdots & \cdots & \cdots & \cdots \\
1 & \varepsilon_{1}^{p-1} & \varepsilon_{2}^{p-1} & \cdots & \varepsilon_{p-1}^{p-1}
\end{array}\right)
$$

in which $\varepsilon_{k}=\exp \left(\frac{2 i k \pi}{p}\right), k=0,1, \cdots, p-1$. Then the linear system equation (6) gives

$$
\left(\mathbf{F}_{B}^{-1} \boldsymbol{\alpha}\right)=\left(\mathbf{F}_{B}^{-1} \mathbf{C}^{-1} \mathbf{F}_{B}\right)\left(\mathbf{F}_{B}^{-1} \mathbf{b}\right)
$$

where

$$
\begin{gathered}
\mathbf{F}_{B}^{-1} \mathbf{C}^{-1} \mathbf{F}_{B}=\operatorname{diag}\left[\begin{array}{llll}
\tilde{\mathbf{R}}_{0}^{-1} & \tilde{\mathbf{R}}_{1}^{-1} & \cdots & \tilde{\mathbf{R}}_{M-1}^{-1}
\end{array}\right], \\
\tilde{\mathbf{R}}=\mathbf{F}_{B} \mathbf{R}^{T}=\left[\begin{array}{llll}
\tilde{\mathbf{R}}_{0} & \tilde{\mathbf{R}}_{1} & \cdots & \tilde{\mathbf{R}}_{M-1}
\end{array}\right]^{T} .
\end{gathered}
$$

By setting

$$
\begin{aligned}
\tilde{\boldsymbol{\alpha}} & =\left[\begin{array}{llll}
\tilde{\boldsymbol{\alpha}}_{0} & \tilde{\boldsymbol{\alpha}}_{1} & \cdots & \tilde{\boldsymbol{\alpha}}_{M-1}
\end{array}\right]^{T}=\mathbf{F}_{B}^{-1} \boldsymbol{\alpha} \\
\tilde{\mathbf{b}} & =\left[\begin{array}{llll}
\tilde{\mathbf{b}}_{0} & \tilde{\mathbf{b}}_{1} & \cdots & \tilde{\mathbf{b}}_{M-1}
\end{array}\right]^{T}=\mathbf{F}_{B}^{-1} \mathbf{b}
\end{aligned}
$$

one obtains

$$
\tilde{\boldsymbol{\alpha}}_{i}=\tilde{\mathbf{R}}_{i}^{-1} \tilde{\mathbf{b}}_{i}, i=0,1, \cdots, M-1
$$

The solution of the linear system (6) yields

$$
\boldsymbol{\alpha}=\mathbf{F}_{B} \tilde{\boldsymbol{\alpha}} .
$$

The above-mentioned matrix solver is called as Fast Toeplitz-type Matrix Solver 1 (FTMS1) in this study.

\section{(b) Type 2: Periodic structure with one-line scatterers}

For solving the periodic structure problems with one-line scatterers, the SBM coefficient matrix $\mathbf{A}$ is a block Toeplitz matrix $\mathbf{T}$ with the form 


$$
\mathbf{A}=\mathbf{T}=\left(\begin{array}{cccc}
\mathbf{T}_{0} & \mathbf{T}_{1} & \cdots & \mathbf{T}_{M-1} \\
\mathbf{T}_{-1} & \mathbf{T}_{0} & \ddots & \mathbf{T}_{M-2} \\
\cdots & \ddots & \ddots & \vdots \\
\mathbf{T}_{1-m} & \mathbf{T}_{2-m} & \cdots & \mathbf{T}_{0}
\end{array}\right),
$$

where each $\mathbf{T}_{i}$ is an $N \times N$ matrix. The number of the unique blocks is $2 M-1$, where $M$ is the number of the scatterers in the periodic structures. Fig. 3 shows a typical periodic structure with one-line scatterers [30], the surface of the kite-shaped scatterer [26] can be represented as

$$
\Gamma=\left\{\left(x_{1}, x_{2}\right): x_{1}=\frac{\cos \varphi+0.65 \cos 2 \varphi-0.65}{6}, x_{2}=0.25 \sin \varphi, 0 \leq \varphi \leq 2 \pi\right\} .
$$

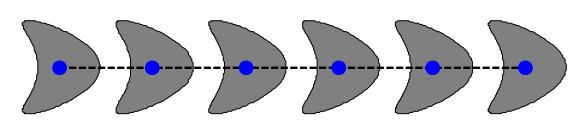

Fig. 3 Schematic configuration of periodic structure with one-line scatterers

According to the property of the block Toeplitz matrix, it can be embedded into a larger block circulant matrix $\mathbf{C}^{\prime}$

$$
\mathbf{C}^{\prime}=\left(\begin{array}{ccccc}
\mathbf{T}_{0} & \mathbf{T}_{1} & \cdots & \mathbf{T}_{-2} & \mathbf{T}_{-1} \\
\mathbf{T}_{-1} & \mathbf{T}_{0} & \mathbf{T}_{1} & \ddots & \mathbf{T}_{-2} \\
\vdots & \mathbf{T}_{-1} & \ddots & \ddots & \vdots \\
\mathbf{T}_{2} & \vdots & \ddots & \ddots & \mathbf{T}_{1} \\
\mathbf{T}_{1} & \mathbf{T}_{2} & \cdots & \mathbf{T}_{-1} & \mathbf{T}_{0}
\end{array}\right) .
$$

Then the first block row $\mathbf{R}^{\prime}=\left[\begin{array}{llllllll}\mathbf{T}_{0} & \mathbf{T}_{1} & \cdots & \mathbf{T}_{M-1} & \mathbf{T}_{1-M} & \mathbf{T}_{2-M} & \cdots & \mathbf{T}_{-1}\end{array}\right]$ of $\mathbf{C}^{\prime}$ needs to be stored and computed, whose computational storage can be reduced to $(2 M-1) / M^{2}$ storage of the full coefficient matrix $\mathbf{T}$. Eq. (6) can be rearranged as follows 


$$
\mathbf{C}^{\prime} \boldsymbol{\alpha}^{\prime}=\mathbf{b}^{\prime} \Leftrightarrow\left(\begin{array}{ll}
\mathbf{C}_{11}^{\prime} & \mathbf{C}_{12}^{\prime} \\
\mathbf{C}_{21}^{\prime} & \mathbf{C}_{22}^{\prime}
\end{array}\right)\left(\begin{array}{l}
\boldsymbol{\alpha} \\
\mathbf{0}
\end{array}\right)=\left(\begin{array}{l}
\mathbf{b} \\
\mathbf{d}
\end{array}\right),
$$

where $\mathbf{C}_{11}^{\prime}=\mathbf{T}$ and $\mathbf{d}=\mathbf{C}_{21}^{\prime} \boldsymbol{\alpha}$. Since both the left-hand and right-hand side vectors $\boldsymbol{\alpha}^{\prime}$ and $\mathbf{b}^{\prime}$ contain unknown vectors $\boldsymbol{\alpha}$ and $\mathbf{d}$, Eq. (15) cannot be solved directly. Here, the simple iterative algorithm in conjunction with the Fast Fourier Transform (FFT) proposed by Ferreira and Dominguez [28] is employed by using the following steps:

i) Insert the known elements of $\mathbf{b}^{\prime}$ into $\mathbf{b}_{k}^{\prime}$, namely, replace $\mathbf{b}_{k}$ by $\mathbf{b}$ and apply the FFT for block circulant matrix to compute the following matrix-vector multiplication

$$
\mathbf{C}^{\prime-1} \mathbf{b}_{k}^{\prime}=\boldsymbol{\alpha}_{k}^{\prime} \Leftrightarrow \mathbf{C}^{\prime-1}\left(\begin{array}{l}
\mathbf{b}_{k} \\
\mathbf{d}_{k}
\end{array}\right)=\left(\begin{array}{l}
\boldsymbol{\alpha}_{k} \\
\mathbf{e}_{k}
\end{array}\right) .
$$

ii) Insert the known elements of $\boldsymbol{\alpha}^{\prime}$ into $\boldsymbol{\alpha}_{k}^{\prime}$, namely, replace $\mathbf{e}_{k}$ by zero vector and apply the FFT for block circulant matrix to compute the following matrix-vector multiplication

$$
\mathbf{C}^{\prime} \boldsymbol{\alpha}_{k}^{\prime}=\mathbf{b}_{k+1}^{\prime} \Leftrightarrow \mathbf{C}^{\prime}\left(\begin{array}{c}
\boldsymbol{\alpha}_{k} \\
\mathbf{0}
\end{array}\right)=\left(\begin{array}{l}
\mathbf{b}_{k+1} \\
\mathbf{d}_{k+1}
\end{array}\right) .
$$

iii) Stop the iteration process if the convergence is achieved with

$$
\left\|\left(\begin{array}{c}
\mathbf{b}_{k+1}-\mathbf{b}_{k} \\
\mathbf{e}_{k}
\end{array}\right)\right\|_{\infty}<\tau_{1},
$$

where $\tau_{1}$ denotes the convergence tolerance for the whole matrix. Otherwise restart the loop.

The above-mentioned matrix solver is called as Fast Toeplitz-type Matrix Solver 2 (FTMS2) in this study.

\section{(c) Type 3: Periodic structure with multiple-line scatterers}

For solving the periodic structure problems with two-line scatterers, the SBM coefficient matrix A is composed of the block Toeplitz submatrices $\mathbf{T}$ with the form 


$$
\mathbf{A}=\left(\begin{array}{cc}
\mathbf{T}^{1} & \mathbf{T}^{2} \\
\mathbf{T}^{3} & \mathbf{T}^{4}
\end{array}\right)
$$

where each $\mathbf{T}^{i}$ is a block Toeplitz matrix having the same form as that of Type2 periodic structure. Fig. 4(a) shows a typical periodic structure with two-line kite-shaped scatterers. Here, the following iterative algorithm is implemented:

i) Set the initial vector $\boldsymbol{\alpha}_{0}=\left[\boldsymbol{\alpha}_{0}^{1}, \boldsymbol{\alpha}_{0}^{2}\right]^{\mathrm{T}}$ determined by solving the equations $\mathbf{T}^{1} \boldsymbol{\alpha}_{0}^{1}=\mathbf{b}^{1}$ and $\mathbf{T}^{4} \boldsymbol{\alpha}_{0}^{2}=\mathbf{b}^{2}-\mathbf{T}^{3} \boldsymbol{\alpha}_{0}^{1}$ in sequence.

ii) Solve the equations $\mathbf{T}^{1} \boldsymbol{\alpha}_{k+1}^{1}=\mathbf{b}^{1}-\mathbf{T}^{2} \boldsymbol{\alpha}_{k}^{2}$ and $\mathbf{T}^{4} \boldsymbol{\alpha}_{k+1}^{2}=\mathbf{b}^{2}-\mathbf{T}^{3} \boldsymbol{\alpha}_{k+1}^{1}$ in sequence.

iii) Stop the iteration process if the convergence is achieved with $\left\|\boldsymbol{\alpha}_{k+1}-\boldsymbol{\alpha}_{k}\right\|_{\infty}<\tau_{2}$, where $\tau_{2}$ denotes the convergence tolerance for the submatrix. Otherwise restart the loop from step ii).

It should be mentioned that the equations with block Toeplitz matrix $\mathbf{T}^{i}$ in steps i) and ii) can be fast calculated by using the FTMS2 mentioned in Section 2.3(b). The above-mentioned matrix solver is called as Fast Toeplitz-type Matrix Solver 3 (FTMS3) in this study.

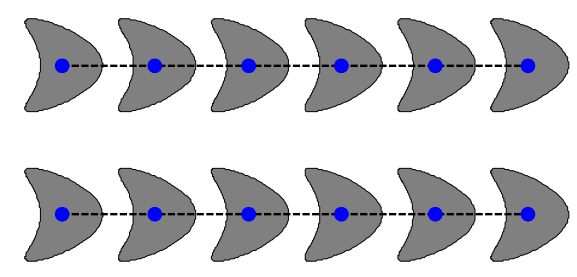

(a) Two periodic arrays of scatterers

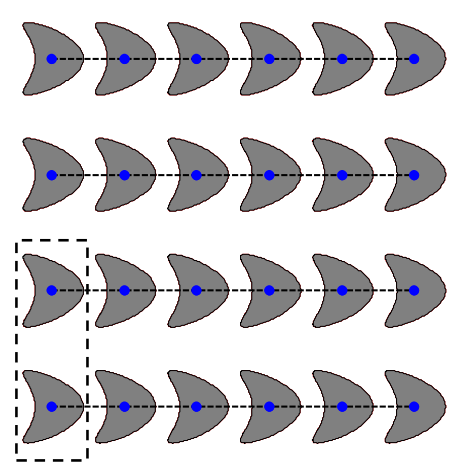

(b) Four periodic arrays of scatterers

Fig. 4 Schematic configuration of periodic structure with multiple-line scatterers (a) two-line arrays, (b) four-line arrays

For solving the periodic structure problems with four-line scatterers as shown in Fig. 4(b), one may consider the two kite-shaped scatterers in one column as a cell 
structure, and then the problem is reduced to the two-line periodic structure problem. Therefore, it can be solved by using the above-mentioned FTMS3.

Finally, it should be mentioned that when the analytical solutions or reference results are not available, the correctness of the present numerical results has been examined by using the following scheme with two steps:

Step (a): Calculating the parameter $\tau_{3}=\left\|\mathbf{b}-\mathbf{A} \boldsymbol{\alpha}_{K}\right\|_{\infty}$. If $\tau_{3}<10^{-10}$, then it may verify that the FTMS runs correctly. Otherwise, reset the initial vector $\boldsymbol{\alpha}_{0}$.

Step (b): Solving the considered problems by using the proposed SBM with two different boundary node numbers $N_{1}, N_{2}\left(2 N_{1} \leq N_{2}\right)$. Then choosing $N K$ random nodes in the computational domain to calculate the relative error

$$
M E=\max _{1 \leq k \leq N K}\left\{\frac{\left|u_{N_{2}}(k)-u_{N_{1}}(k)\right|}{\left|u_{N_{2}}(k)\right|}\right\}
$$

by using the proposed SBM formulation with $N_{1}, N_{2}$. If $M E<10^{-3}$, then it may verify that the present numerical results are correct. Otherwise, reset the boundary node numbers $N_{1}, N_{2}$.

\section{Numerical results}

In this section, the efficiency and accuracy of the proposed method are firstly tested on wave scattering by $2 \mathrm{D}$ circular and $3 \mathrm{D}$ cylinder structures. Then, the comparison between the present SBM results and the reference results [32,33] for water wave scattering by one and two periodic arrays of infinite cylinders is made. Subsequently, the implemented SBM is applied to investigate the differences between the multiple periodic arrays of infinite and finite cylinders under different wave incidence angles. Finally, wave propagation in the periodic structures with $2 \mathrm{D}$ and $3 \mathrm{D}$ irregular scatterers are investigated.

\subsection{Convergence and numerical efficiency analysis}

We firstly investigate the numerical accuracy and efficiency of the proposed method by four benchmark examples. The numerical accuracy is measured by the maximum error $\operatorname{Merr}(u)$ defined as 


$$
\operatorname{Merr}(u)=\max _{1 \leq k \leq N T}|u(k)-\bar{u}(k)|,
$$

where $\bar{u}(k)$ and $u(k)$ are the analytical and numerical solutions at $x_{i}$, respectively, and NT is the total number of the test points in the interested domain. The test points $\left\{\mathbf{y}_{j}\right\}$ are placed on a similar geometric surface to the scatterer with its center at the geometric center gc of the scatterer, which can be defined as follow

$$
\mathbf{y}_{j}=\mathbf{g c}+o d\left[\left(\mathbf{y}_{j}^{0}-\mathbf{g c}\right)\right.
$$

where $\left\{\mathbf{y}_{j}^{0}\right\}$ are the points with a uniform angular distribution on the surface of the scatterer, and $o d$ is the off-distance parameter. Unless otherwise specified, $N K=100$, $N T=200, o d=2, \tau_{1}=10^{-4}$ and $\tau_{2}=10^{-7}$ are used in all the following numerical examples on a personal computer with an Intel Dual-Core of 16GB RAM.

Example 3.1: 2D wave scattering by an infinite circular cylinder with soft $(u=0)$ or rigid $(q=0)$ boundary

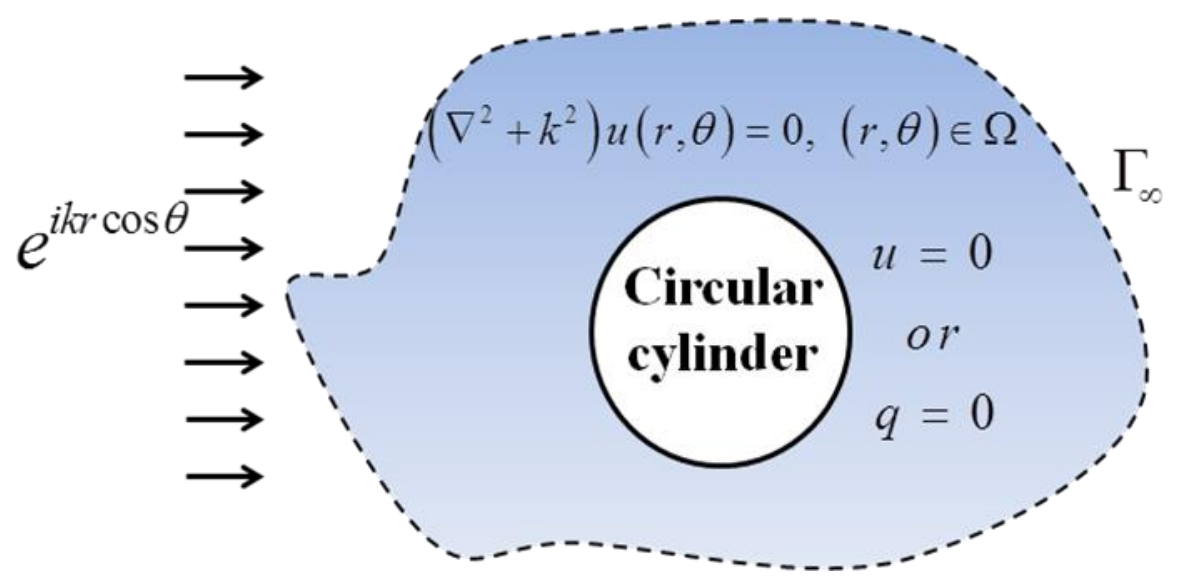

Fig. 3 Sketch of the wave scattering problem by an infinite circular cylinder.

We consider a plane wave $\phi_{I}=e^{i k r \cos \theta_{0}}$ scattered by an infinite circular cylinder (Type 1 structure) as shown in Fig. 3. The analytical solutions of the scattered field $u$ [31] for soft and rigid boundaries are, respectively, $u(r, \theta)=-\frac{J_{0}(k a)}{H_{0}(k a)} H_{0}(k r)-2 \sum_{n=1}^{\infty} i^{n} \frac{J_{n}(k a)}{H_{n}(k a)} H_{n}(k r) \cos n \theta$, for soft boundary, (21a)
$u(r, \theta)=-\frac{J_{0}^{\prime}(k a)}{H_{0}^{\prime}(k a)} H_{0}(k r)-2 \sum_{n=1}^{\infty} i^{n} \frac{J_{n}^{\prime}(k a)}{H_{n}^{\prime}(k a)} H_{n}(k r) \cos n \theta$, for rigid boundary, (21b) 
where the prime denotes differentiation with respect to $k r$, and the analytical solutions are numerically calculated by using the first 150 terms in the above expressions (21). Fig. 4 displays the convergence rate of the present SBM for solving the linear system of equations (6) by using the FFT for 2D wave scattering problem at the normalized wave number $k a=60$. It can be found from Fig. 4 that the present SBM with FTMS1 has a fast convergence rate with the curve-slope 2.6.

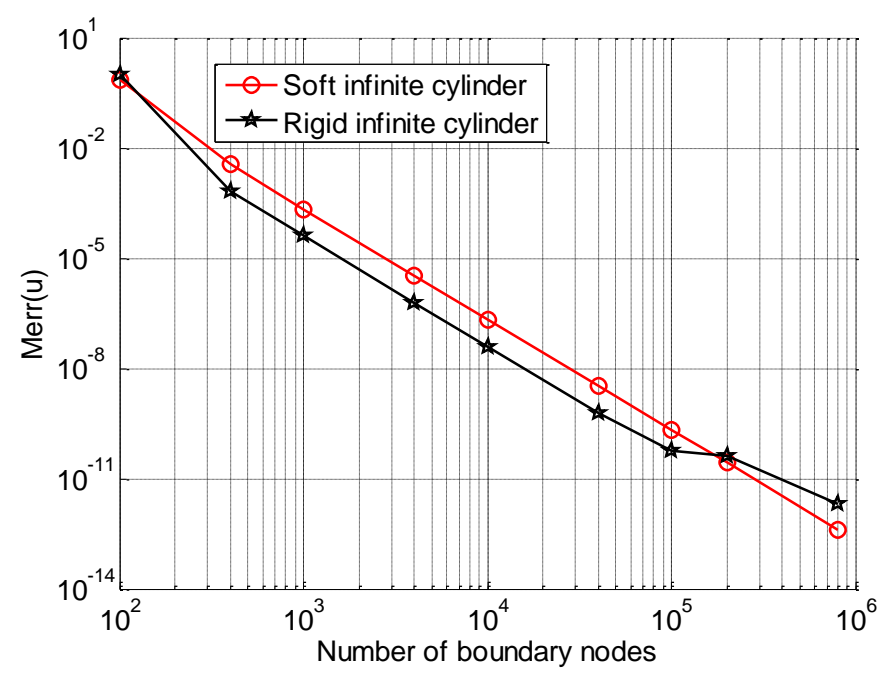

Fig. 4 The convergence rate of the present SBM in Example 3.1.

Example 3.2: $3 \mathrm{D}$ wave radiation by a finite circular cylinder with soft or rigid boundaries

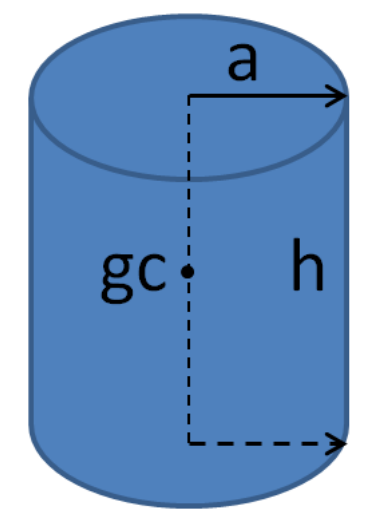

Fig. 5 Sketch of 3D wave radiation problem by a finite circular cylinder

Next we consider the wave radiation problem by a finite circular cylinder (Type 1 structure) as shown in Fig. 5, where the pressure $(u)$ or the normal velocity $(q)$ on the surface of the finite circular cylinder are induced by a point source of the spherical dilatation wave with the unit intensity located at the geometric center. The analytical solution is given by 


$$
u\left(r_{g c}, \theta_{g c}\right)=e^{i k r_{g c}} / r_{g c},
$$

where $\left(r_{g c}, \theta_{g c}\right)$ represents the polar coordinates with the geometric center $g c$ as the origin. Fig. 6 displays the convergence rate of the present SBM for solving the linear system of equations (6) by using the FFT for 3D wave radiation problem with $k a=2$ and $h=2 a$. It can be found from Fig. 6 that the present SBM with FTMS1 has a fast convergence rate with the curve-slope 1.3.

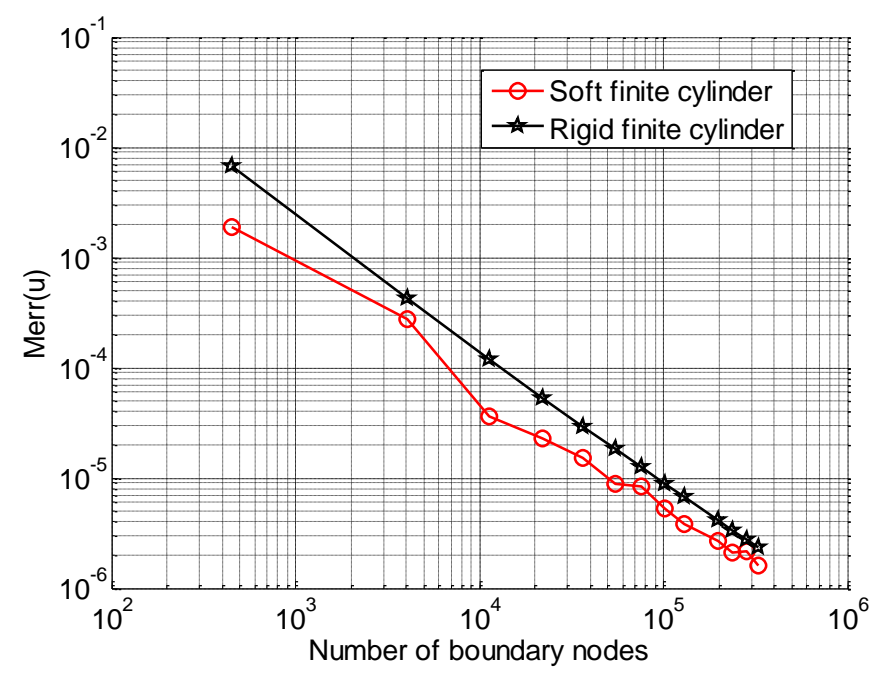

Fig. 6 The convergence rate of the present SBM in Example 3.2.

Table 2. CPU time versus the boundary node number in the present SBM simulation

\begin{tabular}{cccc}
\hline \multicolumn{2}{c}{ 2D case (Example 3.1) } & \multicolumn{2}{c}{ 3D case (Example 3.2) } \\
$N$ & CPU time (s) & $N$ & CPU time (s) \\
\hline 100 & $4.27 \mathrm{E}-05$ & 450 & $1.64 \mathrm{E}-02$ \\
\hline 400 & $5.56 \mathrm{E}-05$ & 4,050 & $1.28 \mathrm{E}-01$ \\
\hline 1,000 & $1.59 \mathrm{E}-04$ & 11,250 & $5.54 \mathrm{E}-01$ \\
\hline 4,000 & $3.70 \mathrm{E}-04$ & 22,050 & $1.86 \mathrm{E}+00$ \\
\hline 10,000 & $8.63 \mathrm{E}-04$ & 36,450 & $4.36 \mathrm{E}+00$ \\
\hline 40,000 & $3.88 \mathrm{E}-03$ & 54,450 & $9.04 \mathrm{E}+00$ \\
\hline 100,000 & $1.17 \mathrm{E}-02$ & 76,050 & $1.68 \mathrm{E}+01$ \\
\hline 200,000 & $2.36 \mathrm{E}-02$ & 101,250 & $2.90 \mathrm{E}+01$ \\
\hline 800,000 & $1.17 \mathrm{E}-01$ & 130,050 & $4.81 \mathrm{E}+01$ \\
\hline$/$ & $/$ & 198,450 & $1.10 \mathrm{E}+02$ \\
\hline$/$ & $/$ & 238,050 & $1.67 \mathrm{E}+02$ \\
\hline$/$ & $/$ & 281,250 & $2.35 \mathrm{E}+02$ \\
\hline$/$ & $/$ & 328,050 & $3.24 \mathrm{E}+02$ \\
\hline
\end{tabular}

Table 2 lists the CPU times versus the number of the boundary nodes in Examples 3.1 and 3.2. It can be found that 2D SBM model with 800,000 boundary 
nodes requires less than 1 second, and 3D SBM with 328,050 boundary nodes requires less than 6 minutes.

\subsection{Numerical comparisons with reference results}

In this section, a numerical comparison between the present SBM and the reference methods proposed by Walker and Taylor [32] and Williams and $\mathrm{Li}$ [33] is given to further verify the efficiency of the present SBM.

Example 3.3: Water wave interaction with one-line 9 impermeable circular cylinders $(q=0)$

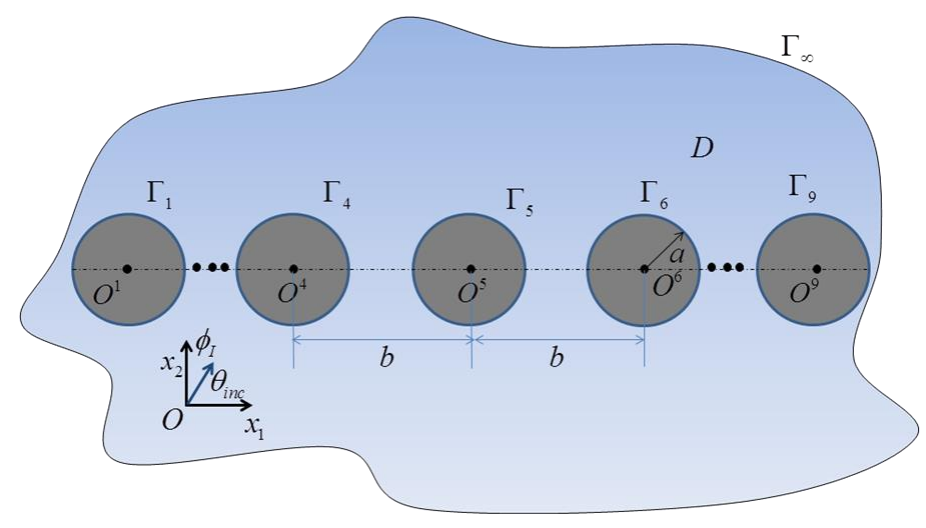

Fig. 7 Sketch of the 2D problem for water wave interaction with one-line 9 circular cylinders

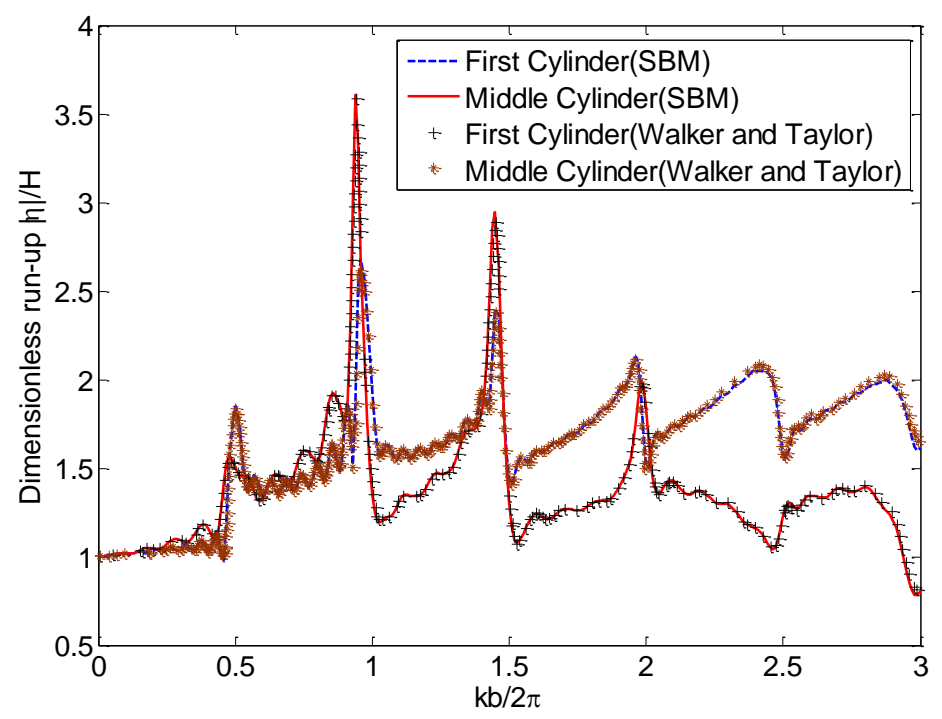

Fig. 8 Dimensionless run-up $|\eta| / H$ on the upstream face of the first and middle cylinders in an array of 9 cylinders plotted against $k b / 2 \pi$. 
First consider the water wave interaction with one-line 9 bottom-mounted impermeable circular cylinders $(q=0)$, which can be simplified to a $2 \mathrm{D}$ problem for an incident plane water wave $\phi_{I}=e^{i k r \cos \theta_{0}}$ scattered by one-line 9 circular rigid obstacles (Type 2 structure) as shown in Fig. 7. For the convenience of comparison with the reference results [32], we set the parameters as follows: radius of the 2D circular cylinders $a=3 / 16$, spacing between the centers of the adjacent cylinders $b=3 / 4$, wave incidence angle $\theta=0$, and boundary node number on the surface of each circular cylinder $N=50$. Fig. 8 presents the dimensionless run-up $|\eta| / H$ on the upstream face of the first and middle cylinders in an array of 9 cylinders with respect to $k b / 2 \pi$, where $\eta=u H / 2$ and $H$ denotes the height of surface water wave. It can be found from Fig. 8 that the results obtained by the present SBM-FTMS2 are in good agreement with the reference results [32].

Example 3.4: Water wave interaction with $2 \times 2$ impermeable circular cylinders $(q=0)$

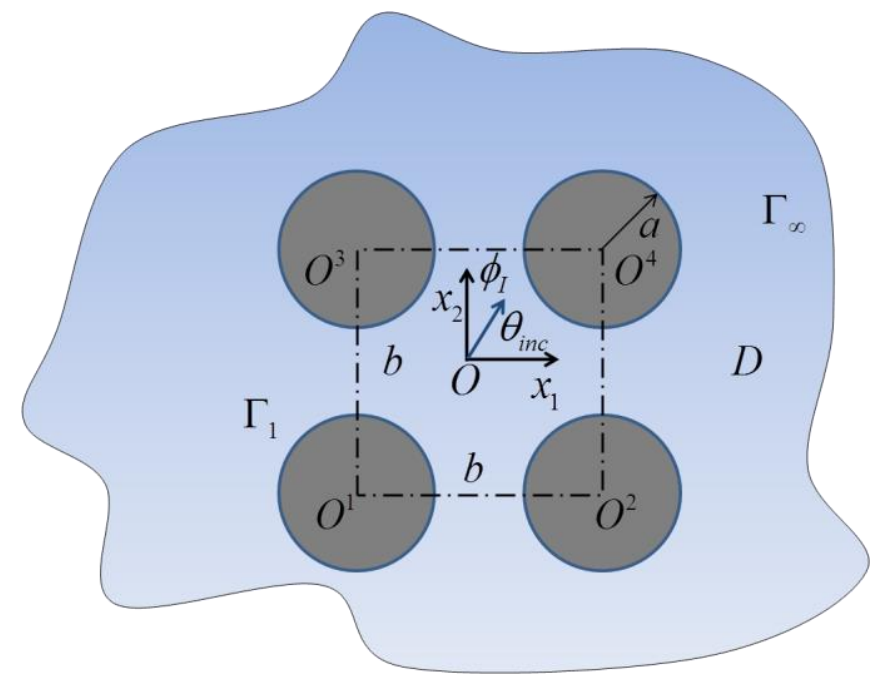

Fig. 9 Sketch of the $2 \mathrm{D}$ problem for water wave interaction with $2 \times 2$ circular cylinders 
(a) Cylinder 1

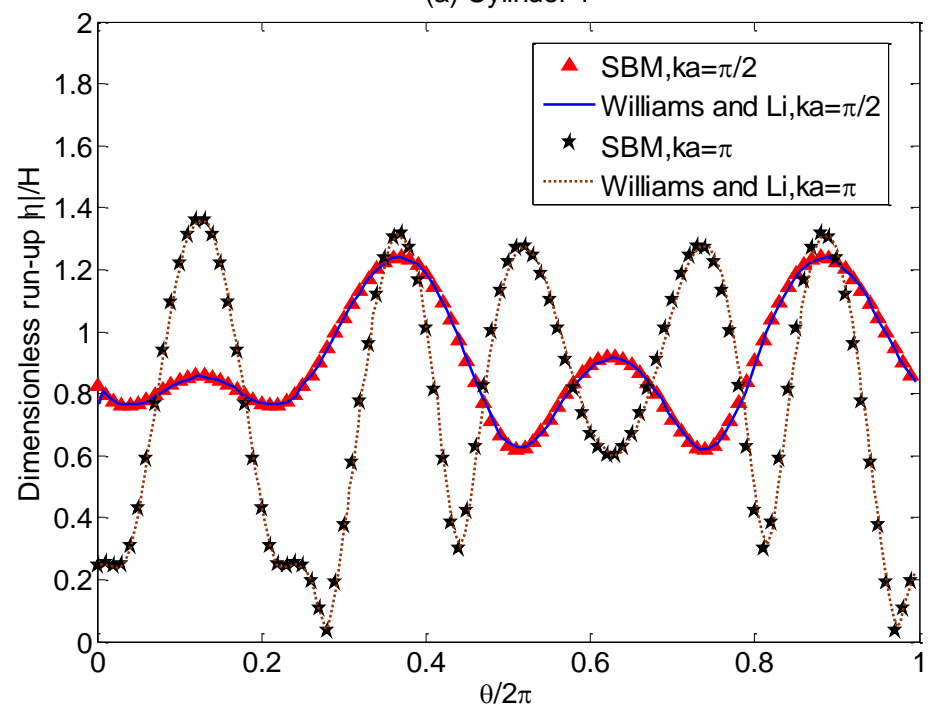

(b) Cylinder 2

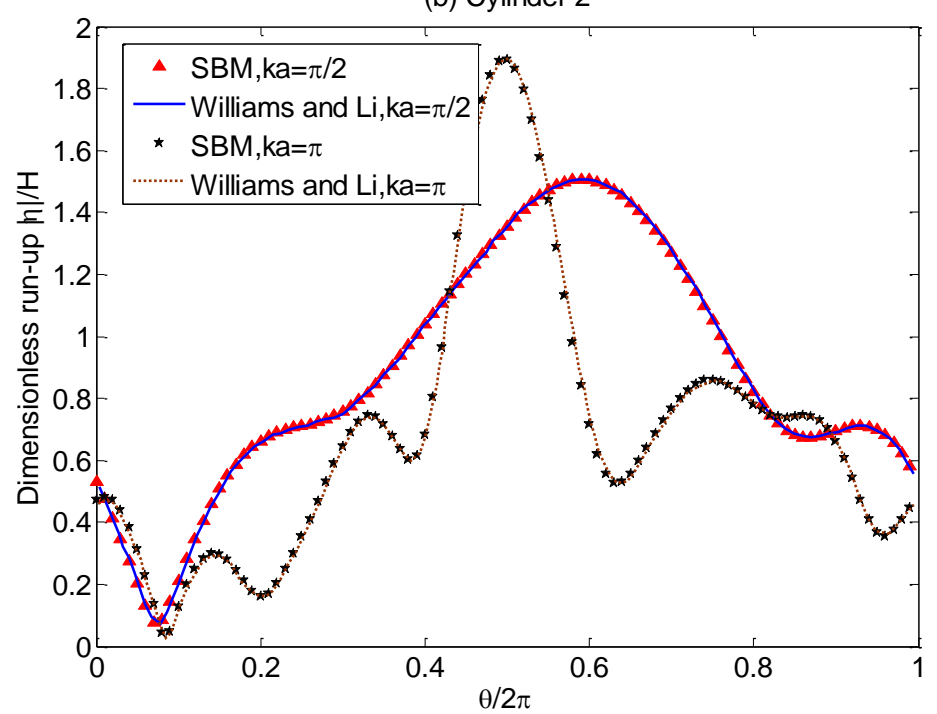

(c) Cylinder 3

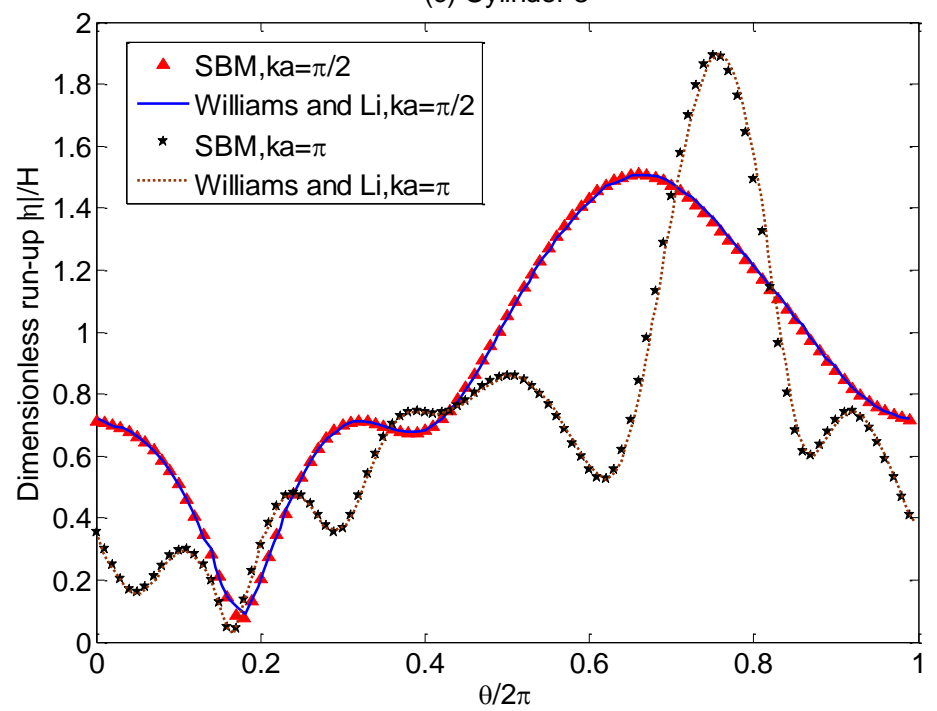




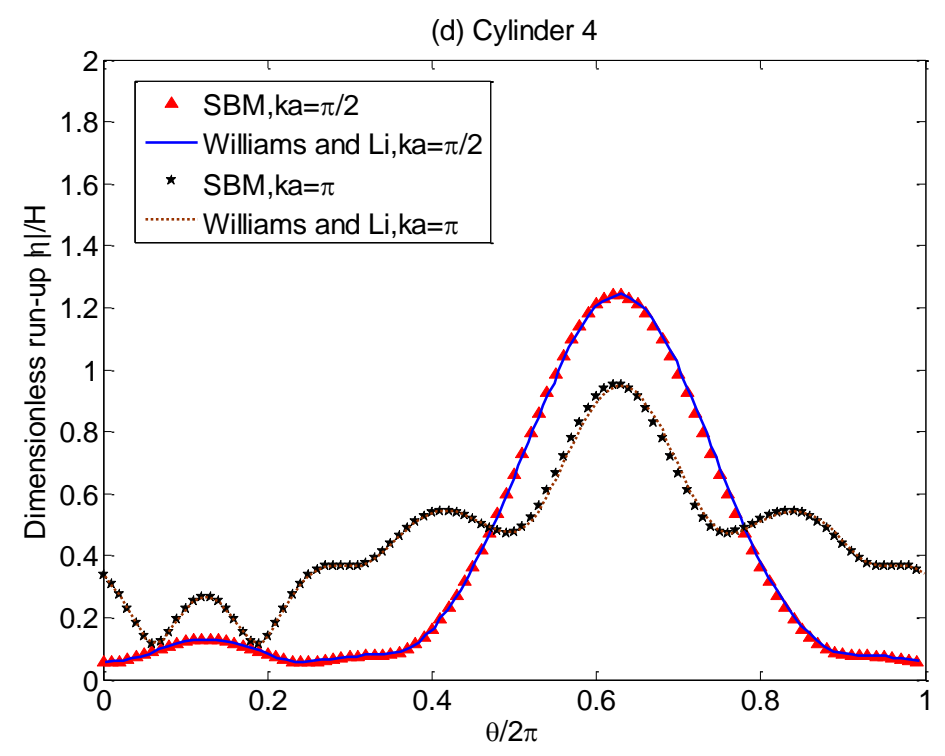

Fig. 10 Dimensionless run-up $|\eta| / H$ on (a) Cylinder 1, (b) Cylinder 2, (c) Cylinder 3, (d) Cylinder 4 in the four-cylinder array with different normalized wave numbers $k a=\pi / 2, \pi$.

Next, we consider the water wave interaction with $2 \times 2$ bottom-mounted impermeable circular cylinders $(q=0)$, which can be simplified to a 2D problem for an incident plane water wave $\phi_{I}=e^{i k r \cos \theta_{0}}$ scattered by $2 \times 2$ circular rigid obstacles (Type 3 structure) as shown in Fig. 9. For the convenience of comparison with the reference results [33], we set the parameters as follows: radius of the $2 \mathrm{D}$ circular cylinders $a=3 / 16$, spacing between the centers of the adjacent cylinders $b=3 / 4$, wave incidence angle $\theta_{0}=\pi / 4$, and boundary node number on the surface of each circular cylinder $N=50$. Fig. 10 presents the dimensionless run-up $|\eta| / H$ on each circular cylinder of the four-cylinder array with different normalized wave numbers $k a=\pi / 2, \pi$. It can be found from Fig. 10 that the results obtained with the present SBM are in good agreement with the reference results. With the increasing normalized wave number $k a$, the dimensionless run-up on the surface of each cylinder becomes more oscillating.

Besides, the following two groups of the specific parameters are set to investigate the near-trapped mode phenomenon: (a) $a=0.4, b=1, k a=4.08482$, 
$\theta_{0}=\pi / 4$; (b) $a=0.4, b=1, k a=5.797, \theta_{0}=5 \pi / 16$. As shown in Fig. 11, the maximum amplitudes appearing on the inner sides of the four cylinders are about 160 and 10 times higher than the incident wave amplitude for the aforementioned two specific parameter settings, respectively.
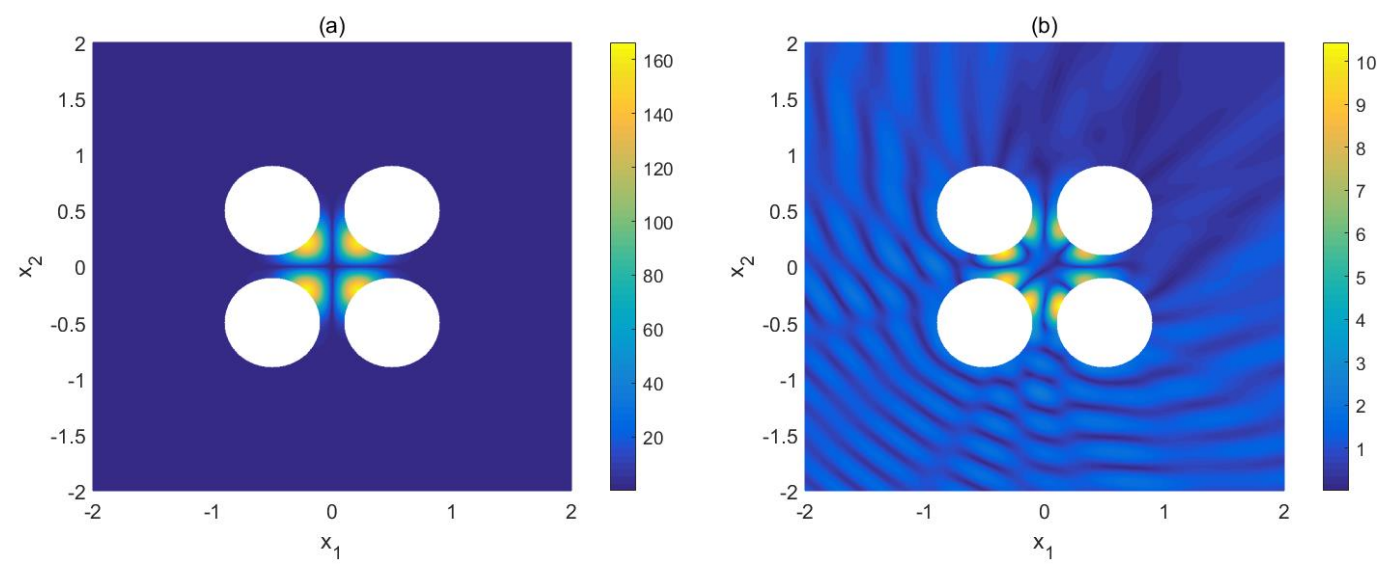

Fig. 11 Near-trapped modes for the four cylinders at the specific parameters: (a) $k a=4.08482$, $\theta_{0}=\pi / 4 ;$ (b) $k a=5.797, \theta_{0}=5 \pi / 16$.

\subsection{Multiple periodic arrays of infinite and finite cylinders}

In this section, we consider the plane wave propagation in structures with multiple periodic arrays of circular cylinders and investigate the effects of the height of the cylinders on the wave propagation behavior around the multiple periodic arrays of the scatterers.

Example 3.5: Wave scattering by $4 \times 4$ impermeable cylinders $(q=0)$

This example considers the acoustic wave scattering problem of a plane wave $u_{I}=e^{i k\left(x_{3} \cos \phi_{0}+\sin \phi_{0}\left(x_{1} \cos \theta_{0}+x_{2} \sin \theta_{0}\right)\right)}$ by $4 \times 4$ impermeable cylinders (Type 3 structure, two cylinders in one column is defined as a cell structure), where $\left(\phi_{0}, \theta_{0}\right)$ denotes the angle of the incident plane wave in the spherical coordinates. When $\phi_{0}=0$ or $\pi$, the plane wave is coming from the positive or negative $x_{3}$-direction. In this case, the schematic configuration of each cylinder is presented in Fig. 5, and the corresponding parameters are set as follows: radius of the circular cylinders $a=3 / 16$, spacing 
between the centers of the adjacent cylinders $b=3 / 8$, non-dimensional wave number $k a=\pi / 2$, angle of the incident wave $\phi_{0}=\pi / 2$, and the boundary node number on the surface of each 3D cylinder $N=288$. It should be mentioned here that the original $3 \mathrm{D}$ problem can be reduced to $2 \mathrm{D}$ problem when the circular rigid cylinders are infinitely long, i.e., $h=$ inf. Then, the boundary node number on the surface of each $2 \mathrm{D}$ circular cylinder is taken as $N=50$. In the numerical verification of the present SBM-FTMS3, we set $N_{1}=144, N_{2}=288$ in $3 \mathrm{D}$ case, and $N_{1}=24, N_{2}=50$ in $2 \mathrm{D}$ case.

Figs. 12-14 show the acoustic pressure amplitude $|u|$ for a $4 \times 4$-cylinder array with the heights (a) $h=\inf$; (b) $h=3$; (c) $h=2$; (d) $h=1$ on the $x_{3}=0$ plane for various wave incidence angles $\left(\theta_{0}=0, \pi / 6, \pi / 4\right)$. It can be observed from Figs. 12-14 that the acoustic pressure amplitude for the normal wave incidence $\theta_{0}=0$ is symmetrical with respect to the line $x_{2}=0$, and the acoustic pressure amplitude for the wave incidence angle $\theta_{0}=\pi / 4$ is symmetrical about the line $x_{1}=x_{2}$.
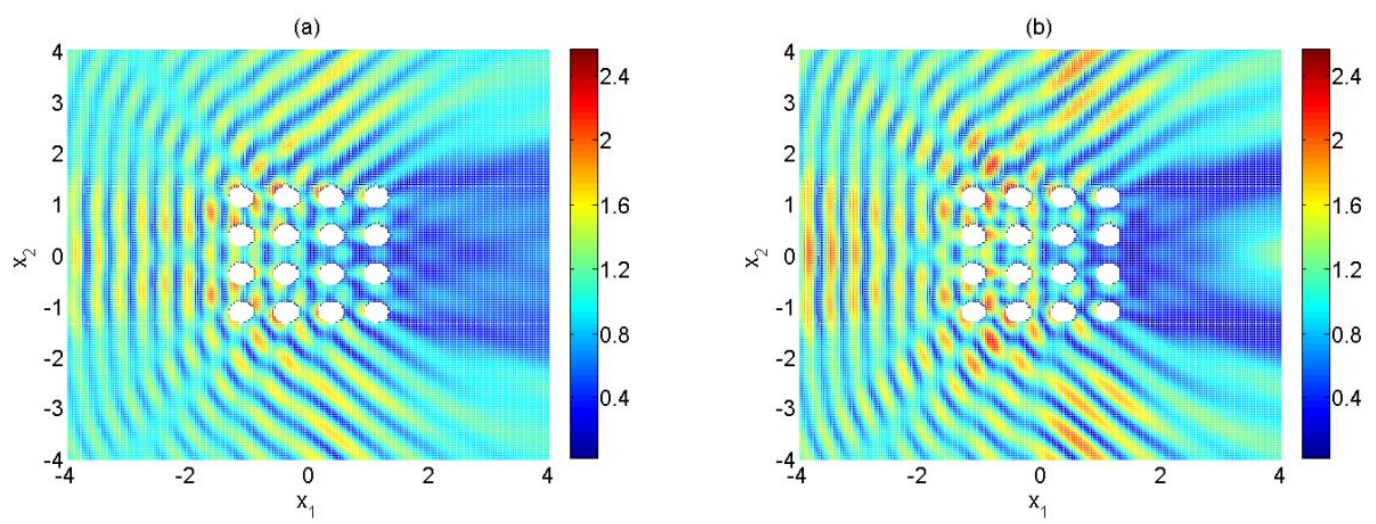
(c)

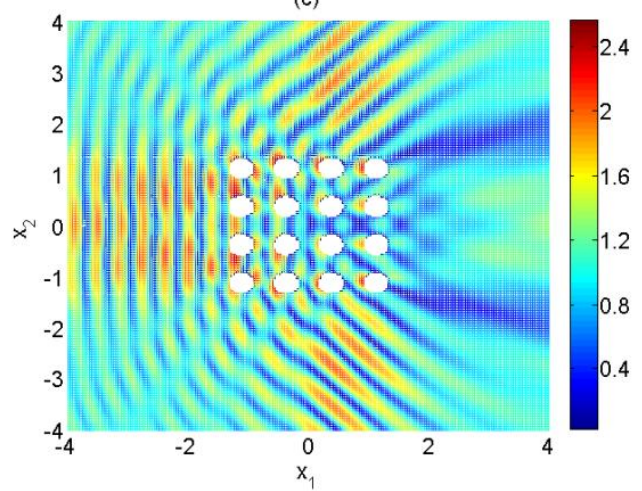

(d)

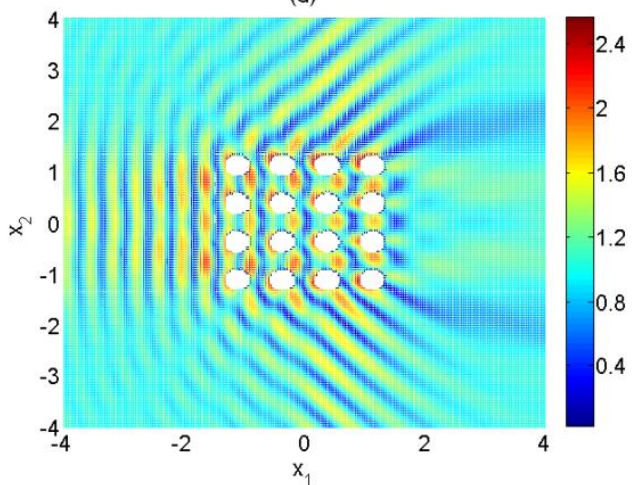

Fig. 12 Acoustic pressure amplitude $|u|$ for the $4 \times 4$-cylinder array with different heights (a) $h=\inf$; (b) $h=3$; (c) $h=2$; (d) $h=1$ on the $x_{3}=0$ plane $\left(\theta_{0}=0\right)$.

(a)

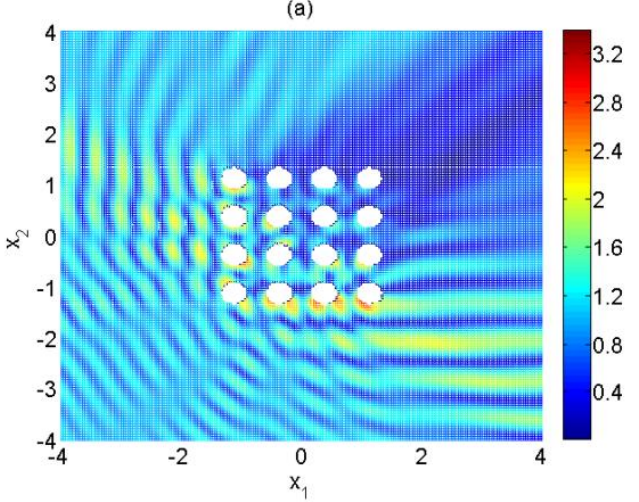

(c)

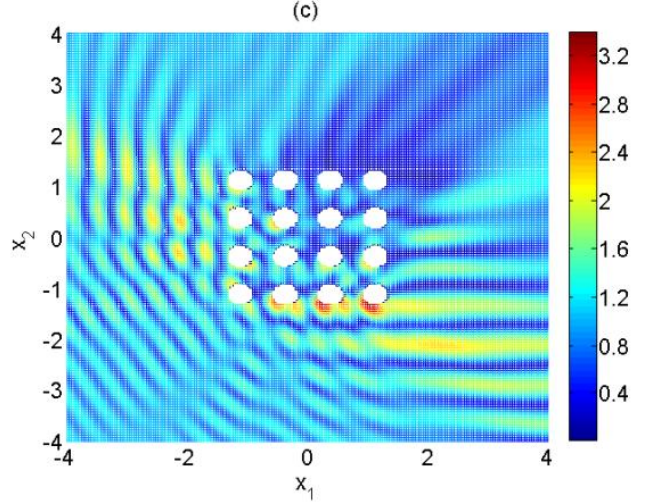

(b)

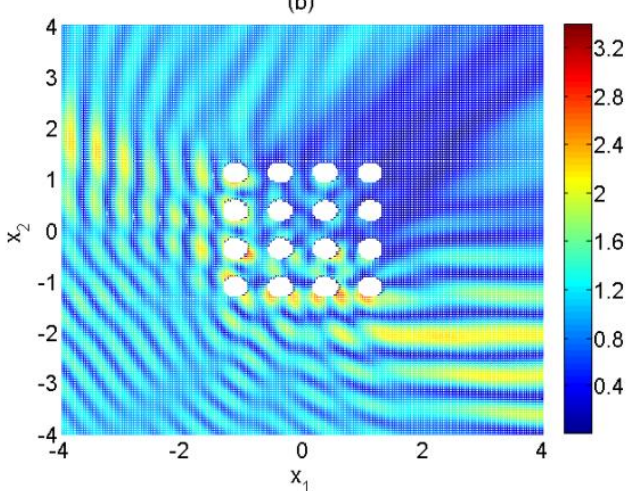

(d)

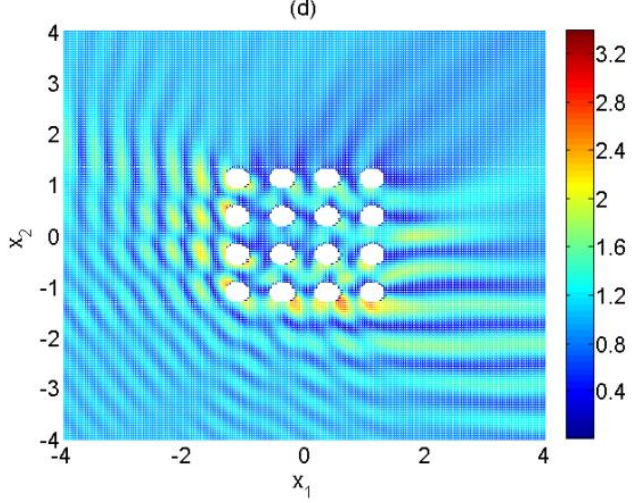

Fig. 13 Acoustic pressure amplitude $|u|$ for the $4 \times 4$-cylinder array with different heights (a) $h=\inf$; (b) $h=3$; (c) $h=2$; (d) $h=1$ on the $x_{3}=0$ plane $\left(\theta_{0}=\pi / 6\right)$. 

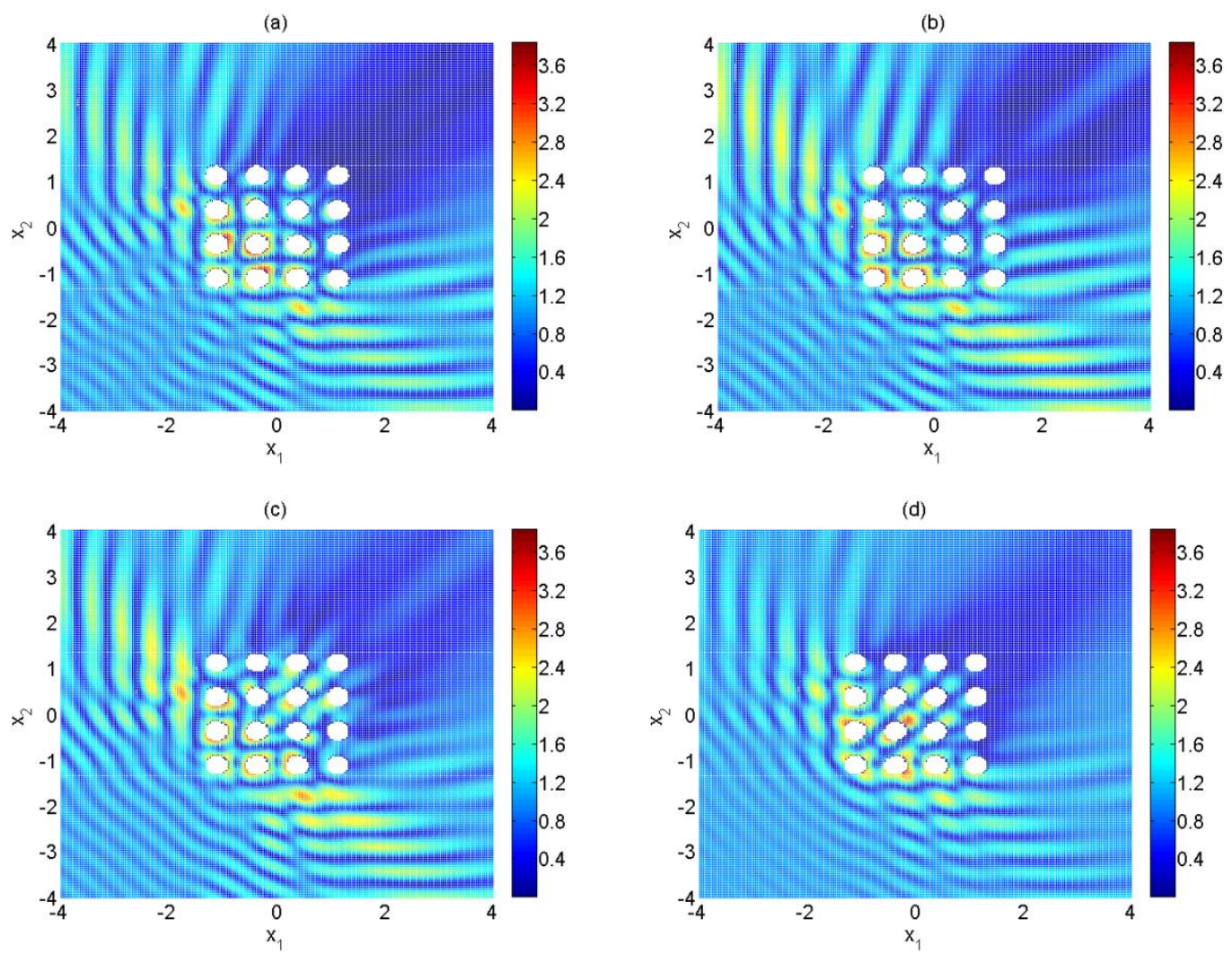

Fig. 14 Acoustic pressure amplitude $|u|$ for the $4 \times 4$-cylinder array with different heights (a) $h=\inf$; (b) $h=3$; (c) $h=2$; (d) $h=1$ on the $x_{3}=0$ plane $\left(\theta_{0}=\pi / 4\right)$.

In comparison with the $3 \mathrm{D}$ models for different heights of the cylinders, 2D models provide the smallest acoustic pressure amplitudes in the nearby downstream region of the $4 \times 4$-cylinder array. This is because there is no diffraction by the top surface of the scatterers in the 2D models. Furthermore, with the increasing height of the cylinder $h$, the acoustic pressure amplitude is decreasing in the nearby downstream region of the $4 \times 4$-cylinder array. It reveals that the periodic structures with a regular array of the scatterers can block the wave propagation at certain frequency or non-dimensional wave number $k a$, which can be used for vibration control and noise reduction in engineering applications.

\subsection{Structures with periodic arrays of $2 \mathrm{D}$ and $3 \mathrm{D}$ irregular scatterers}

In this section, the wave propagation simulation for structures with periodic arrays of 2D and 3D irregular scatterers are presented.

Example 3.6: Wave scattering by one-line 7 infinite $m$-shaped impermeable cylinders 


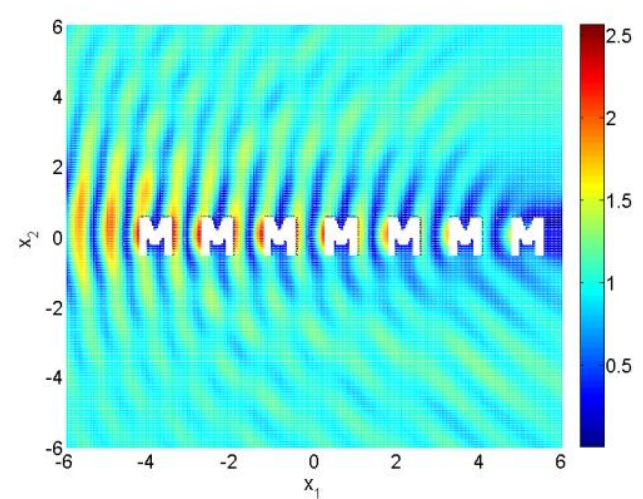

(a) $\operatorname{SBM}$ results $\left(\theta_{0}=0\right)$

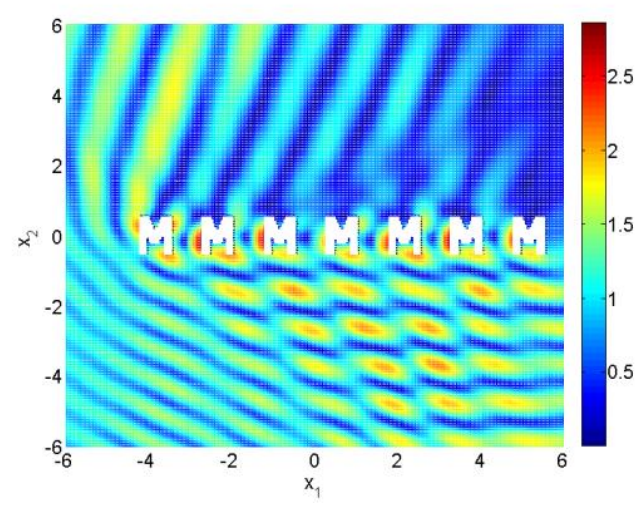

(c) $\operatorname{SBM}$ results $\left(\theta_{0}=\pi / 4\right)$

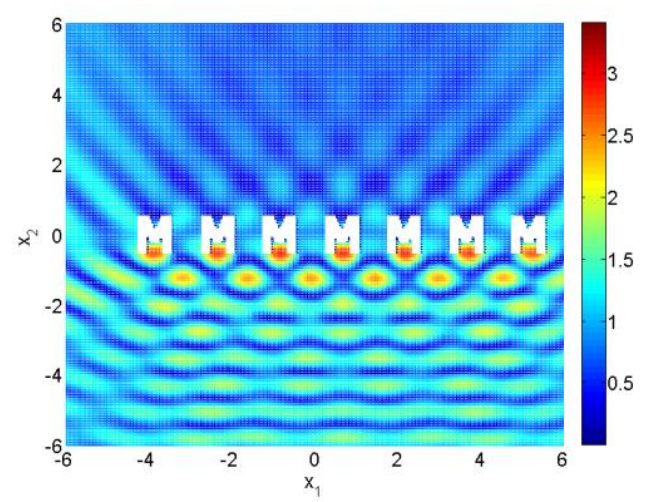

(e) $\operatorname{SBM}$ results $\left(\theta_{0}=\pi / 2\right)$

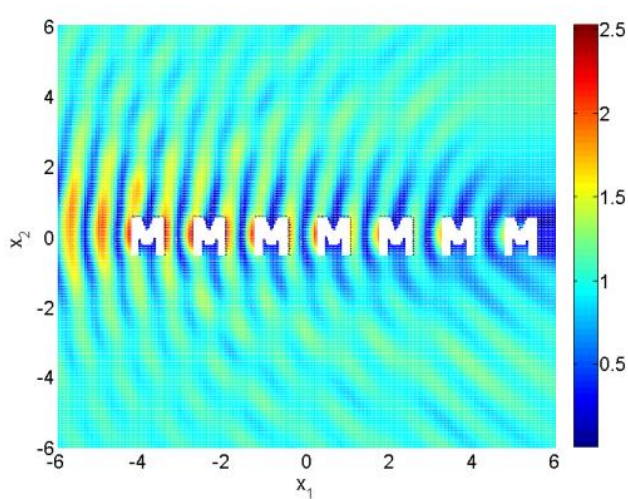

(b) $\operatorname{COMSOL}$ results $\left(\theta_{0}=0\right)$

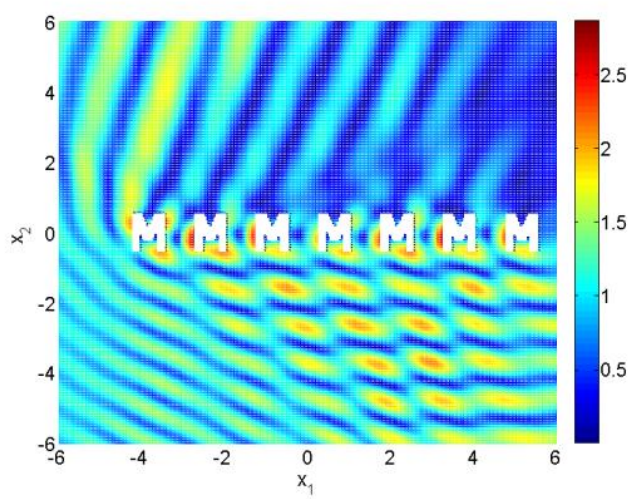

(d) $\operatorname{COMSOL}$ results $\left(\theta_{0}=\pi / 4\right)$

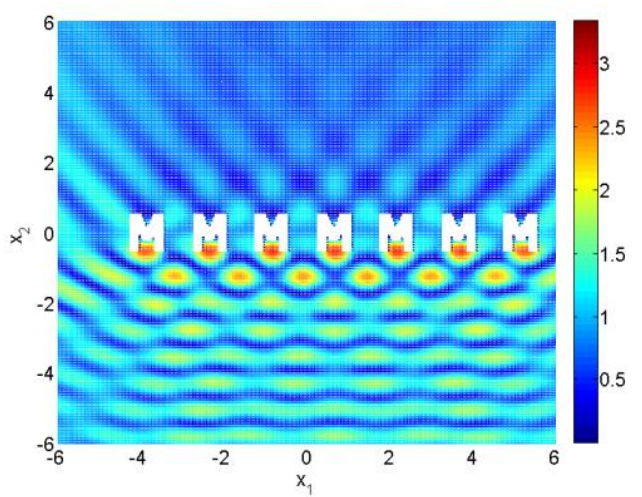

(f) COMSOL results $\left(\theta_{0}=\pi / 2\right)$

Fig. 15 Acoustic pressure amplitude $|u|$ for one-line $7 \mathrm{~m}$-shaped cylinder array at different wave incidence angle $\theta_{0}$ with the wave number $k=4.1888$ by using the present SBM-FTMS2 ( $7 \times 345$ boundary nodes) and the COMSOL software (26613 domain elements and 1031 boundary elements), (a) SBM results $\left(\theta_{0}=0\right)$, (b) COMSOL results $\left(\theta_{0}=0\right)$, (c) $\operatorname{SBM}$ results $\left(\theta_{0}=\pi / 4\right),(\mathrm{d})$ $\operatorname{COMSOL}$ results $\left(\theta_{0}=\pi / 4\right)$, (e) SBM results $\left(\theta_{0}=\pi / 2\right)$, (f) $\operatorname{COMSOL}$ results $\left(\theta_{0}=\pi / 2\right)$.

We consider the acoustic wave interaction with one-line 7 infinite $\mathrm{m}$-shaped 
impermeable cylinders, which can be simplified to a 2D scattering problem of an incident plane acoustic wave $\phi_{I}=e^{i k r \cos \theta_{0}} \quad$ scattererd by one-line 7 rigid $\mathrm{m}$-shaped obstacles (Type 2 structure). The corners of the middle m-shaped obstacle are $(0.3,0.5),(0.475,0.5),(0.65,-0.05),(0.825,0.5),(1,0.5),(1,-0.5),(0.9,-0.5),(0.9,0.4)$, $(0.7,-0.2),(0.6,-0.2),(0.4,0.4),(0.4,-0.5),(0.3,-0.5)$. The distance between the centers of two adjacent m-shaped obstacles is 3/2. Fig. 15 depicts the acoustic pressure amplitude $|u|$ for one-line $7 \mathrm{~m}$-shaped cylinder array at different wave incidence angle $\theta_{0}=0, \pi / 4, \pi / 2$ with the wave number $k=4.1888$ by using the present SBM-FTMS2 $(7 \times 345$ boundary nodes $)$ and the COMSOL software (26613 domain elements and 1031 boundary elements). It can be found from Fig. 14 that the present SBM-FTMS2 with fewer boundary nodes can provide the similar numerical results to the COMSOL software.

Example 3.7: Wave scattering by $20 \times 20$ infinite kite-shaped impermeable cylinders We consider the acoustic wave interaction with $20 \times 20$ infinite kite-shaped impermeable cylinders, which can be simplified to a 2D scattering problem of an incident plane acoustic wave $\phi_{I}=e^{i k r \cos \theta_{0}}$ scattererd by $20 \times 20$ rigid kite-shaped obstacles (Type 3 structure, 10 kite-shaped obstacles in one column is defined as a cell structure). Fig. 16 depicts the acoustic pressure amplitude $|u|$ for the $20 \times 20$ kite-shaped-cylinder array at the wave incidence angle $\theta=\pi / 4$ and the non-dimensional wave number $k a=\pi / 2$ by using 100 boundary nodes on the surface of each kite-shaped scatterer. In the numerical verification of the present SBM-FTMS3, we set $N_{1}=50, N_{2}=100$. It should be mentioned that it cannot obtain the correct results when $20 \times 20$ rigid kite-shaped obstacles are considered as Type 2 structure for taking 20 kite-shaped obstacles in one column as a cell structure. It reveals that the proposed SBM with the FTMS3 is more stable than the FTMS2 in the simulation of multiple-line periodic structures. 


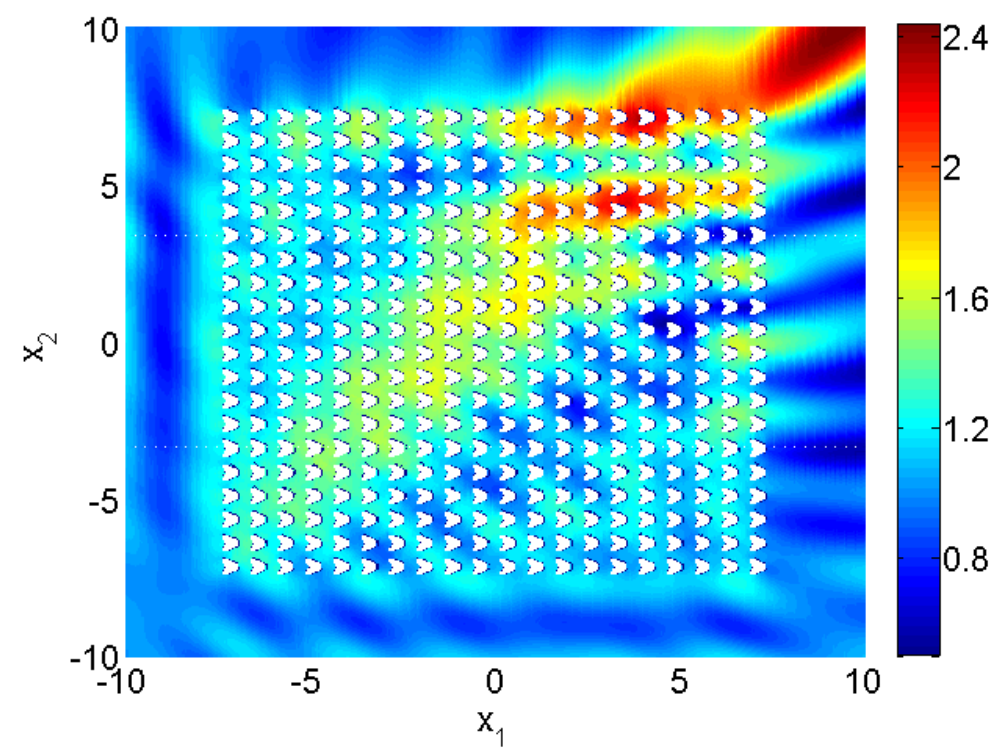

Fig. 16 Acoustic pressure amplitude $|u|$ for the $20 \times 20$ kite-shaped cylinder array at the wave incidence angle $\theta_{0}=\pi / 4$ and the non-dimensional wave number $k a=\pi / 2$ by using 100 boundary nodes on the surface of each kite-shaped scatterer.

Example 3.8: Wave scattering by $4 \times 4$ top-shaped impermeable axisymmetric scatterers

This example considers the scattering of a plane time-harmonic wave $u_{I}=e^{i k\left(x_{3} \cos \phi_{0}+\sin \phi_{0}\left(x_{1} \cos \theta_{0}+x_{2} \sin \theta_{0}\right)\right)} \quad$ by the $4 \times 4$ top-shaped impermeable axisymmetric scatterers (Type 3 structure, 2 top-shaped axisymmetric scatterers in one column is defined as a cell structure). Fig. 17 shows the acoustic pressure amplitude $|u|$ for the $4 \times 4$ top-shaped impermeable axisymmetric scatterers at the wave incidence angle $\phi_{0}=\pi / 2, \theta_{0}=\pi / 4$ and the non-dimensional wave number $k a=\pi / 2$ by using 600 boundary nodes on the surface of each top-shaped axisymmetric scatterer. In the numerical verification of the present SBM-FTMS3, we set $N_{1}=288, N_{2}=600$. 


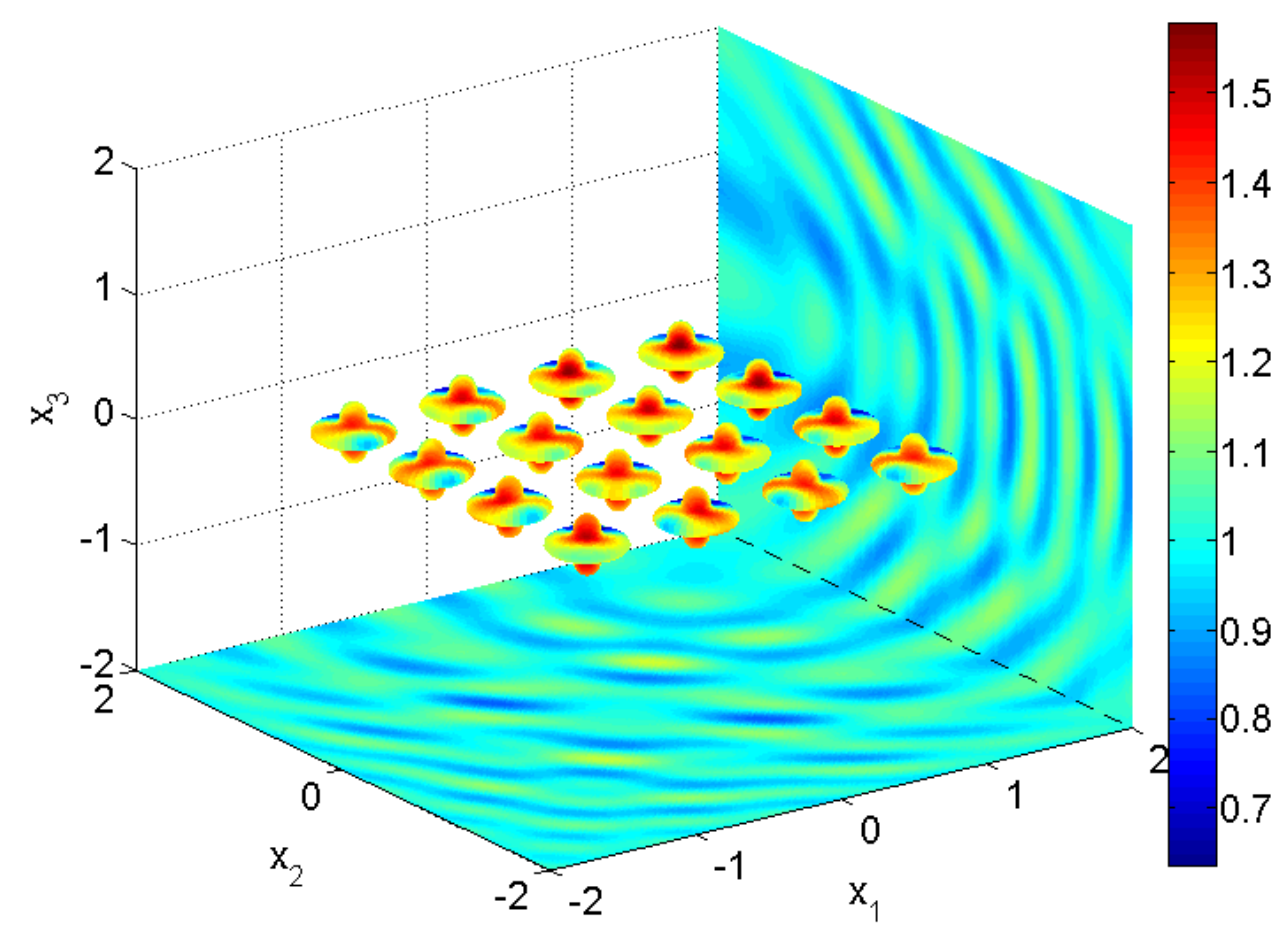

Fig. 17 Acoustic pressure amplitude $|u|$ for the $4 \times 4$ top-shaped impermeable axisymmetric scatterers at the wave incidence angle $\phi_{0}=\pi / 2, \theta_{0}=\pi / 4$ with the non-dimensional wave number $k a=\pi / 2$.

\section{Conclusions}

This paper presents the singular boundary method (SBM) in conjunction with the fast Toeplitz-type matrix solvers (FTMS) for the acoustic wave propagation analysis in periodic structures with a non-dimensional wavenumber $k d<60$. According to the types of the SBM resultant matrix generated by different types of the periodic structures, three fast Toeplitz-type matrix solvers are implemented. The efficiency and accuracy of the proposed FTMS-SBM are tested on wave scattering problems by $2 \mathrm{D}$ circular and 3D cylindrical scatteres.

Numerical results verify that the present SBM provides satisfactory solutions and converges to the exact solutions with the increasing boundary node number (the curve-slope is 2.6 in $2 \mathrm{D}$ and 1.3 in $3 \mathrm{D}$ ). Then numerical comparison shows that the present SBM results are in good agreement with the reference results for the water 
wave scattering problem by one-line 9 infinite cylinders and $2 \times 2$-array infinite cylinders. Different heights or lengths of the cylinders are considered to show its effects on the acoustic pressure amplitude around the multiple periodic arrays of the scatterers. With the increasing height of the cylinders, the acoustic pressure amplitudes will decrease in the nearby downstream region of the multiple periodic arrays of the scatterers. Finally, the numerical results show that the present SBM can simulate the acoustic wave interaction with the periodic array of $2 \mathrm{D}$ and $3 \mathrm{D}$ irregular objects up to 800,000 boundary nodes (2D) or 300,000 boundary nodes (3D) on a personal computer with an Intel Dual-Core of 16GB RAM. Besides, numerical results show that the proposed SBM-FTMS3 is more stable than the SBM-FTMS2 in the simulation of multiple-line periodic structures.

Overall, it is concluded that the proposed FTMS-SBM could be considered as a competitive alternative for wave propagation analysis in periodic structures after further extensive numerical and theoretical study. As for the theoretical error analysis of the SBM, it is a nontrivial task. So far, only one related work [34] has been reported, which gives the error bounds of the SBM for potential problems. The theoretical error analysis of the SBM for wave propagation problems is still under our intensive investigation. Besides, it is worth noting that as the first step, we only considered wave propagation by $2 \mathrm{D}$ and $3 \mathrm{D}$ periodic structures with impenetrable boundary conditions in this study. The proposed FTMS-SBM for wave propagation by periodic structures with penetrable boundary conditions is under intense study and will be reported in a subsequent paper.

\section{Acknowledgements}

The authors thank the anonymous reviewers of this article for their very helpful comments and suggestions to significantly improve the academic quality of this article. The work reported in this paper is supported by the Natural Science Foundation of China (Grant Nos. 11772119, 11572111, 11302069), the Chinese Postdoctoral Science Foundation (Grant Nos. 2014M561565, 2015T80492), the 
Foundation for Open Project of the State Key Laboratory of Acoustics (Grant No. SKLA201509), the Fundamental Research Funds for the Central Universities (Grant No. 2016B06214), the 111 Project (Grant No. B12032), Qing Lan Project. The second and fourth authors would like to thank the China Scholarship Council (CSC) and the German Academic Exchange Service (DAAD) for the financial support.

\section{References}

[1] C. Droz, C. Zhou, M.N. Ichchou, J.P. Laine, A hybrid wave-mode formulation for the vibro-acoustic analysis of 2D periodic structures, Journal of Sound \& Vibration, 363 (2015) 285-302.

[2] Z. Guo, M. Sheng, J. Pan, Effect of boundary conditions on the band-gap properties of flexural waves in a periodic compound plate, Journal of Sound \& Vibration, 395 (2017) 102-126.

[3] J. Huang, M. Ruzzene, S. Chen, Analysis of in-plane wave propagation in periodic structures with Sierpinski-carpet unit cells, Journal of Sound \& Vibration, 395 (2017) 127-141.

[4] D.J. Moreau, L.A. Brooks, C.J. Doolan, Broadband trailing edge noise from a sharp-edged strut, Journal of the Acoustical Society of America, 129 (2011) 2820-2829.

[5] Z.L. Ji, Boundary element analysis of a straight-through hybrid silencer, Journal of Sound \& Vibration, 292 (2006) 415-423.

[6] Z. Yan, C. Zhang, Y. Wang, Wave propagation and localization in randomly disordered layered composites with local resonances, Wave Motion, 47 (2010) 409-420.

[7] J. Mei, Z. Liu, C. Qiu, Multiple-scattering theory for out-of-plane propagation of elastic waves in two-dimensional phononic crystals, Journal of Physics Condensed Matter, 17 (2005) 3735-3757.

[8] R.M. Orris, M. Petyt, A finite element study of harmonic wave propagation in periodic structures, Journal of Sound \& Vibration, 33 (1974) 223-236.

[9] F.L. Li, Y.S. Wang, C. Zhang, G.L. Yu, Boundary element method for band gap calculations of two-dimensional solid phononic crystals, Engineering Analysis with Boundary Elements, 37 (2013) 225-235.

[10] M.S. Kushwaha, P. Halevi, L. Dobrzynski, B. Djafari-Rouhani, Acoustic band structure of periodic elastic composites, Physical Review Letters, 71 (1993) 2022.

[11] B. Nennig, E. Perrey-Debain, J.D. Chazot, The method of fundamental solutions for acoustic wave scattering by a single and a periodic array of poroelastic scatterers, Engineering Analysis with Boundary Elements, 35(2011)1019-1028.

[12] E. Perrey-Debain, J. Trevelyan, P. Bettess, Plane wave interpolation in direct collocation boundary element method for radiation and wave scattering: numerical aspects and applications, Journal of Sound and Vibration, 261 (2003) 839-858.

[13] W. Chen, Singular boundary method: A novel, simple, meshfree, boundary collocation numerical method. (in Chinese), Acta Mechanica Solida Sinica, 30 (2009) 592-599.

[14] Z.-J. Fu, W. Chen, Y. Gu, Burton-Miller-type singular boundary method for acoustic radiation and scattering, Journal of Sound and Vibration, 333 (2014) 3776-3793.

[15] J. Li, W. Chen, Z. Fu, L. Sun, Explicit empirical formula evaluating original intensity factors of singular boundary method for potential and Helmholtz problems, Engineering Analysis with Boundary Elements, 73 (2016) 161-169. 
[16] Z.J. Fu, W. Chen, J.T. Chen, W.Z. Qu, Singular boundary method: Three regularization approaches and exterior wave applications, CMES: Computer Modeling in Engineering \& Sciences, 99 (2014) 417-443.

[17] I. Gonzalez, F.S.D. Adana, F. Catedra, Application of the multilevel fast multipole method to the analysis of conformed multilayered periodic structures, in: IEEE Antennas and Propagation Society International Symposium, 2007, pp. 1849-1852.

[18] C.J. Zheng, H.F. Gao, L. Du, H.B. Chen, C. Zhang, An accurate and efficient acoustic eigensolver based on a fast multipole BEM and a contour integral method, Journal of Computational Physics, 305 (2016) 677-699.

[19] S. Kurz, O. Rain, S. Rjasanow, The adaptive cross-approximation technique for the 3D boundary-element method, IEEE Transactions on Magnetics, 38 (2002) 421-424.

[20] N. Mishra, J. Vondrejc, J. Zeman, A comparative study on low-memory iterative solvers for FFT-based homogenization of periodic media, Journal of Computational Physics, 321 (2016) 151-168.

[21] A.T. Tran, H.L. Quang, Q.C. He, Computation of the size-dependent elastic moduli of nano-fibrous and nano-porous composites by FFT, Composites Science \& Technology, 135 (2016) 159-171.

[22] S.C. Jiang, B. Teng, Y. Gou, D.Z. Ning, A precorrected-FFT higher-order boundary element method for wave-body problems, Engineering Analysis with Boundary Elements, 36 (2012) 404-415.

[23] J. Xiao, W. Ye, Y. Cai, J. Zhang, Precorrected FFT accelerated BEM for large-scale transient elastodynamic analysis using frequency-domain approach, International Journal for Numerical Methods in Engineering, 90 (2012) 116-134.

[24] R.M. Gray, Toeplitz and circulant matrices : A review, Communications and Information Theory, 2 (2001) 155-239.

[25] N. Levinson, The Wiener RMS error criterionin filter design and prediction, J. Math. Phys., 25 (1947) 261-278.

[26] S. Chandrasekaran, A.H. Sayed, A fast stable solver for nonsymmetric Toeplitz and quasi-Toeplitz systems of linear equations, SIAM Journal on Matrix Analysis \& Applications, 19 (2000) 107-139.

[27] R. Chan, X. Jin, An Introduction to Iterative Toeplitz Solvers, Society of Industrial and Applied Mathematics, 2007.

[28] P.J.S.G. Ferreira, M.E. Domínguez, Trading-off matrix size and matrix structure: Handling Toeplitz equations by embedding on a larger circulant set, Digital Signal Processing, 20 (2010) 1711-1722.

[29] M. Karimi, P. Croaker, N. Kessissoglou, Boundary element solution for periodic acoustic problems, Journal of Sound \& Vibration, 360 (2016) 129-139.

[30] Y. Liu, A.H. Barnett, Efficient numerical solution of acoustic scattering from doubly-periodic arrays of axisymmetric objects, Journal of Computational Physics, 324 (2016) 226-245.

[31] J.T. Chen, C.T. Chen, P.Y. Chen, I.L. Chen, A semi-analytical approach for radiation and scattering problems with circular boundaries, Computer Methods in Applied Mechanics and Engineering, 196 (2007) 2751-2764.

[32]Walker D A G, Taylor R E. Wave diffraction from linear arrays of cylinders. Ocean Engineering, 32(2005) 2053-2078.

[33] A.N. Williams, W. Li, Water wave interaction with an array of bottom-mounted surface-piercing porous cylinders, Ocean Engineering, 27 (2000) 841-866.

[34] J.P. Li, W. Chen, Y. Gu, Error bounds of singular boundary method for potential problems. 
Numerical Methods for Partial Differential Equations, 33 (2017) 1987-2004. 